\title{
WestVirginiaUniversity
}

THE RESEARCH REPOSITORY @ WVU

Graduate Theses, Dissertations, and Problem Reports

2005

\section{Estimation of image quality factors for face recognition}

Akintunde A. Akinbola

West Virginia University

Follow this and additional works at: https://researchrepository.wvu.edu/etd

\section{Recommended Citation}

Akinbola, Akintunde A., "Estimation of image quality factors for face recognition" (2005). Graduate Theses, Dissertations, and Problem Reports. 4132.

https://researchrepository.wvu.edu/etd/4132

This Thesis is protected by copyright and/or related rights. It has been brought to you by the The Research Repository @ WVU with permission from the rights-holder(s). You are free to use this Thesis in any way that is permitted by the copyright and related rights legislation that applies to your use. For other uses you must obtain permission from the rights-holder(s) directly, unless additional rights are indicated by a Creative Commons license in the record and/ or on the work itself. This Thesis has been accepted for inclusion in WVU Graduate Theses, Dissertations, and Problem Reports collection by an authorized administrator of The Research Repository @ WVU. For more information, please contact researchrepository@mail.wvu.edu. 


\section{Estimation of Image Quality Factors for Face Recognition}

by

Akintunde A. Akinbola

Thesis Submitted to the

College of Engineering and Mineral Resources

at West Virginia University

in partial fulfillment of the requirements

for the degree of

Master of Science
in
Electrical Engineering

Natalia A. Schmid, D.SC., Chair

Lawrence Hornak, Ph.D.

Xin Li, Ph.D.

Lane Department of Computer Science and Electrical Engineering

Morgantown, West Virginia

2005

Keywords: Quality factors, Estimation, Lighting, Eigenphase, Eigenface 


\author{
Abstract \\ Estimation of Quality Factors for Face Recognition \\ by \\ Akintunde A. Akinbola \\ Masters of Science in Electrical Engineering \\ West Virginia University \\ Natalia A. Schmid, D.SC., Chair
}

Over the past few years, verification and identification of humans using biometric has gained attention of researchers and of the public in general. Face recognition systems are used by the public and the government and are applied in different facets of life including security, identification of criminals and identification of terrorists. Because of the importance of these applications, it is of great necessity that face recognition systems be as accurate as possible. Some research has shown that image quality degrades the performance of face recognition systems. Most previous research has focused on designing algorithms for face recognition that deal or compensate a single effect such as blur, lighting conditions, pose, and emotions. In this thesis we identify a number of factors influencing recognition performance and conduct an extensive study of the effects of image quality factors on recognition performance and discuss methods to estimate this quality factors. 


\section{Acknowledgements}

I would like to thank everyone who helped me get through this thesis. My sincere thanks goes to my advisor, Dr Schmid for all her effort, assistance, and patience and believe in me. Her work ethics, motivation and push have helped me complete this thesis. Her willingness and availability to answer all my questions and give me feedback is greatly appreciated.

I am also grateful to my colleagues, Yash N. Shah, Nathan D. Kalka, Vivekanand Dorairaj, and Jinyu Zuo for all their assistance and support. I also would like to thank Dr. Hornak and Dr. Xin Li for accepting to be a member of my committee on a short notice.

Finally, I am thankful to the entire Lane Department of Computer Science and Electrical Engineering and all the faculty and staff members for all the knowledge they have transferred to me this past few years. 


\title{
Contents
}

\begin{abstract}
Acknowledgements

iii

Contents iv

List of Figures $\quad$ vi

List of Tables vi vi
\end{abstract}

1. Introduction $\quad \mathbf{1}$

1.1 Biometrics Technology.............................................. 1

1.2 Face Recognition.................................................... 2

1.3 Face Quality Evaluation............................................. 4

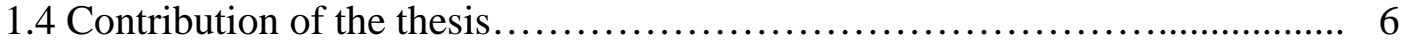

1.5 Organization of the thesis.......................................... 6

2. Effect of Quality Factors on Performance............................. 7

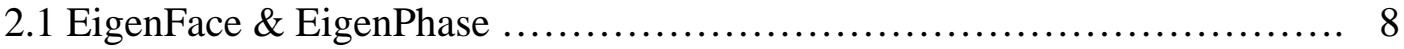

2.2 Defocused Blur........................................................ 13

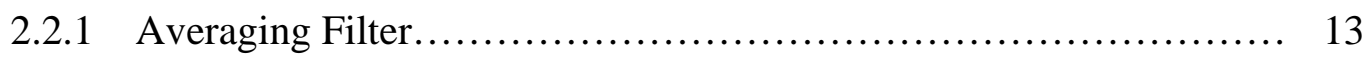

2.3 Motion Blur..................................................... 14

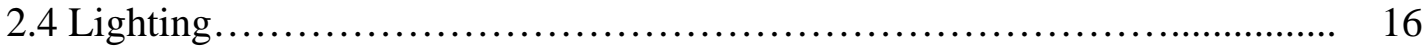

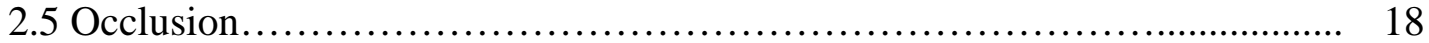

2.6 Cropping............................................................ 18

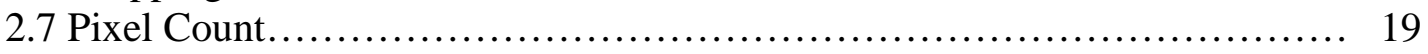

2.8 Facial Expression............................................... 20

3. Synthetic study.................................................... 21

3.1 YALE Database................................................... 22

3.2 WVU Database.................................................. 27

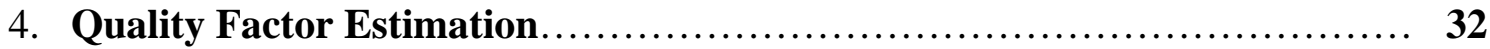

4.1 Motion Blur........................................................ 32

4.1.1 Estimation of direction of blur................................. 34

4.1.2 Estimation of Blur strength.................................. 37

4.2 Defocus Blur........................................................ 39

4.3 Lighting.............................................................. 40 
4.3.1 Histogram................................................. 40

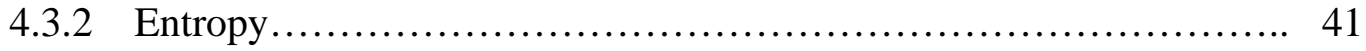

4.3.3 Direction of External Light..................................... 42

4.3.4 Degree of degradation.......................................... 43

4.3.5 Experimental Results........................................ 45

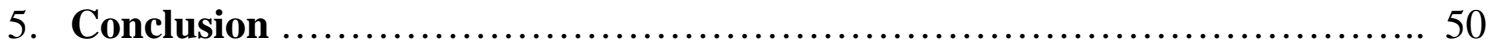

6. Future Work ..................................................... 51

References........................................................... 52 


\section{List of Figures}

1.1 Traditional Face recognition System.................................... 3

$2.1 \quad$ Eigenfaces........................................................... 11

2.2 Defocus face images.................................................. 13

2.3 Averaging Filter....................................................... 14

2.4 Motion Blurred face images.......................................... 15

2.5 Fourier transform of filter used to simulate synthetic motion blur............... 16

2.6 Linear Filter.......................................................... 16

$2.7 \quad$ Face Images at varying lighting conditions.................................... 17

2.8 Occluded face images................................................... 18

2.9 Cropped face images................................................. 19

2.10 Face images at varying pixel count..................................... 19

2.11 Face images with varying expressions.................................... 20

3.1 Model for Recognition Performance study............................... 21

3.2 Matching score for defocus blur........................................... 22

3.3 Matching score for motion blur....................................... 23

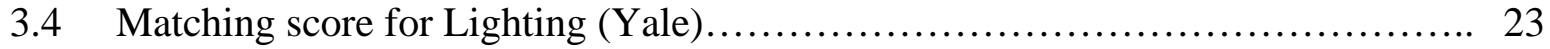

3.5 Matching score for Cropping (Yale) ......................................... 24

3.6 Matching score for Pixel count (Yale).................................... 25

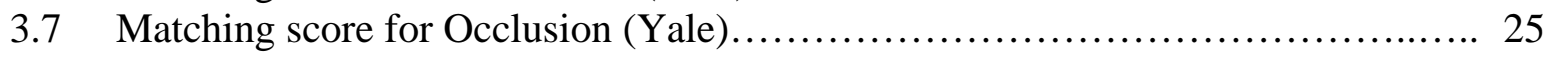

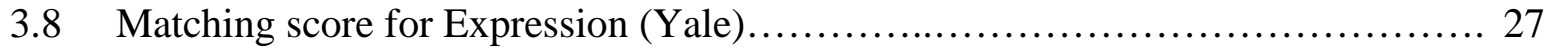

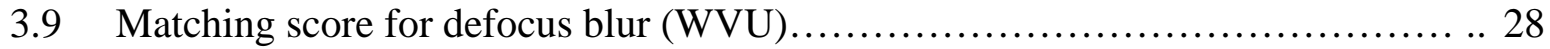

3.10 Euclidean score for motion blur (WVU) ................................. 28

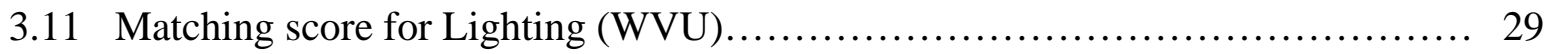

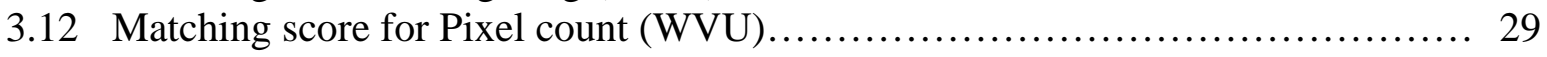

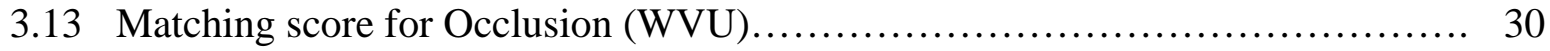

$4.1 \quad$ Blur estimation model................................................. 35

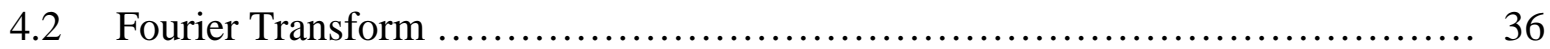

4.3 Fourier transform of Image and corresponding filter response.................. 37

4.4 Block diagram for Motion blur estimation.................................. 38

4.5 Histogram of an images with poor brightness and contrast..................... 41

4.6 Image expose to external lighting from the left of the subject................... 43

4.7 Histogram of four equally divided segments of a face image $\ldots \ldots \ldots \ldots \ldots \ldots \ldots \ldots . \ldots 45$

4.8 Experimental result for defocus blur using method $1 \ldots \ldots \ldots \ldots \ldots \ldots \ldots \ldots \ldots \ldots . \ldots 47$

4.9 Experimental results for lighting variation (contrast) .................... 48

4.10 ROC curves for lighting variation (contrast) $\ldots \ldots \ldots \ldots \ldots \ldots \ldots \ldots \ldots \ldots \ldots \ldots . \ldots \ldots$

\section{List of Tables}

4.1 Experimental result for motion blur...................................... 46

4.2 Experimental result for defocus blur using method $2 \ldots \ldots \ldots \ldots \ldots \ldots \ldots \ldots \ldots \ldots . \ldots \ldots$ 


\section{Chapter 1}

\section{Introduction}

\subsection{Biometric Technology}

Biometrics is a field of computer vision that has gained recognition and become an important area of research over the years. Theoretically, Biometrics is the automatic recognition of a person using distinguishing traits [7]. In practice Biometrics involve comparing a newly captured image to a set of preprocessed images with similar features in an already existing database. Biometric technology is used in different various applications. Biometrics can operate in two modes; verification and identification [7]. Verification is a one-to-one match of images while identification is a one-to-many match.

Every human being has features different from others. Among this features are the features of the face, iris, fingerprint and hand. Along with the distinct characteristic of these features, these features remain unchanged for a long period of time. These characteristics are the basis for feature recognition of human beings.

This thesis focuses on recognition using facial features (Face Recognition).

The need for a more accurate and efficient recognition system increases everyday with rising security demand [30]. Majority of the traditional and common verification and identification methods are not as dependable and reliable as they used to be when initially introduced. Pin number and Passwords for example can be stolen or guessed. With Biometric 
technology, it is much more difficult to steal an identity. With Biometric technology, you do not have to remember passwords, pin numbers; or carry access cards

\section{Applications of Biometrics}

Including, but not limited to the following:

- Security

- Criminal Identification

- Terrorist Identification

- Member verification

- Fraud Reduction and detection

\subsection{Face Recognition}

Research in face recognition has grown over the years, mainly because of the increasing need for a more reliable and dependable security system. Facial recognition deals with recognizing the human face by recording the spatial geometry of distinguishing features of the face [7]. It employs distinct features of the face. Facial recognition is used for verification and identification related applications. Verification involves a one-to-one match and identification, a one-to-many match. An advantage of facial recognition over the other recognition technologies is that it does not require the subject's cooperation or awareness which makes it highly suitable for security related applications such as criminal or terrorist 
identification. Facial recognition is applied in different facets of life, including the identification of missing children. Face recognition involves four main steps (see fig 1.1):

I. Capturing and localizing a face image (Face detection)

II. Face Alignment

III. Extracting the features of the face, and

IV. Matching (Comparing this features with another template or a pre existing database depending on the task at hand and making a decision).

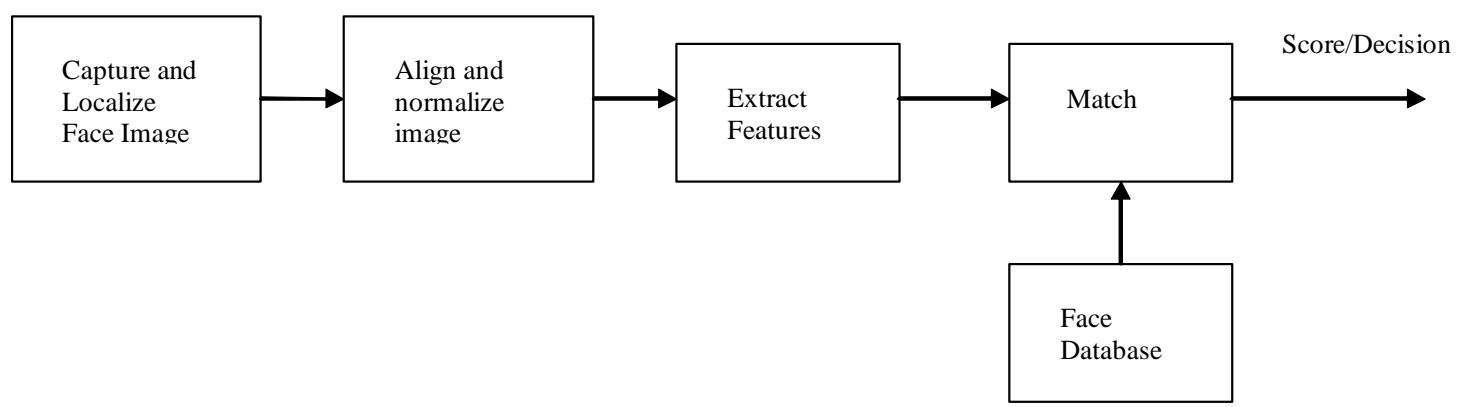

Figure 1.1: Traditional Face recognition System

Capturing is the actual acquisition of the image using a static camera or a video system, while localization is performed to segment the face from the background. Face alignment is a process of normalizing the face to create symmetry (especially in orientation and distance). Upon localization and alignment, the features of the resulting face image are extracted to be used for matching. The final step, matching, involves comparing the extracted features of face images with features extracted from face images stored in the database for verification or identification purposes. 


\subsection{Face Quality Evaluation}

The concept of matching relies on the difference between user templates. The degree of variation dictates the decision. Matching scores are computed based on these variations and compared to a threshold value for decision making. The rationale is based on the fact that under controlled environment intra user distance-based matching scores (genuine scores) are generally lesser in value than inter user distance-based matching scores (imposter score) because of higher inter user variation (variation in the features of two human faces). For Face recognition we want a high inter user variation and a minute intra user variation. A matching error will result in a situation where the intra user variation is higher than the inter user variation (that is the imposter score is lower than the genuine score). Intra user variation is caused by changes in a user's physiological characteristics, acquisition environment or a combination of both.

Two images of the same user acquired at different times may appear different due to changes in the physiological characteristics of the user - the user could wear his/her hair differently, smile in one capture and frown in the other. Image appearance could also vary depending on the acquisition environment - two images taken under different environmental conditions will appear different. The capture device or user could also cause image variations. Variations due to changes in physiological characteristics are mostly visible to the human eye, while variations due to changes in acquisition environment may or may not be visible to the human eye depending on the degree of variations. These variations could seriously degrade the performance of the matching process. 
The factors that cause these intra class variations are defined as the quality factors of an image. Prior research has shown that indeed the performance of face recognition algorithms is affected by one or more of these quality factors [30], but to our knowledge there has been no extensive study on the assessment of these quality factors for face recognition.

Prior research shows that the following listed factors affect the performance of face recognition [23] [32].

- The relative motion between the target (face) and the camera (motion blur),

- Poor camera focus (Defocus blur)

- Lighting/illumination

- Occlusion

- Image resolution

- Cropping, and

- Expression, and

- Pose

These factors could seriously degrade recognition performance and hence it is necessary to detect and compensate for them. Facial recognition is applied in situations that are very critical; hence it is of great necessity that recognition (matching) be as accurate as possible. In lieu of this captured images need to be assessed for quality to determine if it is 
good enough (quality wise) to be used for recognition or fairly poor that it can be compensated for or corrected or very poor that it should not be used for recognition.

As of the moment, there is no algorithms invariant to every single one of these factors. Some algorithms are invariant to some factors but not all. It is important to evaluate the degree of effect of each of this quality factors on recognition to decide if correction is feasible or not.

We also may define local quality (quality of facial skin texture) and global (overall image quality) quality factors.

\subsection{Contribution of the Thesis}

This thesis, using two Face database performs an extensive study on the assessment of these image quality factors and also discusses algorithms that can be used in detect and estimate some of these factors - Lighting, Motion blur and Defocus Blur.

\subsection{Organization of the Thesis}

In Chapter 2, we list and characterize the quality factors affecting face recognition, describe the synthetic simulation of these quality factors and carry out performance study. Results of the performance study are discussed in Chapter 3, while Chapter 4 focuses on detecting and estimating quality factors. Conclusion and Future work are provided in chapter 6 and 7 , respectively. 


\section{Chapter 2}

\section{Effect of Quality Factors on Recognition Performance}

This section discusses the effects that the image quality factors listed in Section 1.3 produce on the performance of face recognition systems. We describe the steps taken to reach our conclusion. Of all the databases available to us, the Yale face database was the most suitable for our study. The database contained single light source face images for 39 users cropped and aligned, each with varying pose and illumination conditions. The database was sufficient to carry out performance study on lighting and expression image quality factors under real life conditions. Other factors, - motion blur, defocus blur, occlusion, pixel count, and cropping were synthetically simulated. We also studied the effect of these quality factors on face recognition using the WVU database.

Each quality factor was studied separately using two common face recognition algorithms - Eigenface and Eigenphase [3] [24] [25]. The results are discussed in Chapter 3.

The effect of each image quality factor was studied in a similar manner. For each user in the Yale and WVU database, we select visually good images and store them as a separate database to be used for training. For each quality factor -motion blur, defocus blur, occlusion, 
lighting, pixel count, and cropping, except for expression, we selected from each user another visually good image, synthetically degraded it at different degrees and stored them as separate databases to be used for testing . Section 2.2 to 2.7 describes how these quality factors were simulated. For lighting, we conducted the effect of brightness using synthetically degraded images of the WVU database and the effect of external lighting using the real degraded images of the Yale database. For expressions, we used the real images in the Yale database. As previously mentioned, the non-degraded visually good images were used for training and the degraded set of images used for testing. Templates created from the training set are compared with those of the test images using the Euclidean distance metric. Two graphs were generated for each quality factor studied; one for Eigenface and the other for Eigenphase. Each graph shows the error bar plot of the genuine score and the error bar plot of the imposter score of matching. Genuine scores are the scores generated from comparing intra user face images (images of the same user) and imposter scores are scores generated from comparing inter user images (images of different users). The Euclidean distance is plotted as a function of the strength of the quality factor being studied. The plot shows the effect on performance as the degree of degradation of the quality factor increases. The standard deviation of the error bar plot depicts the overlap of the genuine score and imposter score. The more the overlap, the worse the performance.

\subsection{Eigenface \& Eigenphase}

Some of the common algorithms used for face recognition are the Eigenface [3] [24] [25], Eigenphase [24], feature analysis [34] [35] and neural network [33] [35] .This thesis 
focuses on the Eigenface and Eigenphase algorithms. Eigenfaces were first introduced by Sirovich and Kirby (1987) [14] and are simply a set of eigenvectors derived from the estimated covariance matrix of the human face. Eigenface is defined on wikipedia [4] as a set of eigenvectors derived from the covariance matrix of the probability distribution of the high-dimensional vector space of possible faces of human beings used to classify faces based on general facial patterns (features). The algorithm works by constructing a smaller feature image (Eigenfaces) from the features extracted from a set of trained face images using the principal components analysis (PCA) [13].

A template (weights of each eigenface for reconstruction the face) is created for each image in the training set from a combination of these eigenfaces and used in matching for identification and verification.

Prior to extracting the face features, the images are resampled to have same resolution and are converted to column vectors.

\section{Steps to calculate Eigenface}

Following [3],

Step 1: Each face image is converted into a vector $\Gamma_{n}$ of length $\mathrm{N}$ (image width $\times$ image height). The faces constituting the training set $\Gamma$ are prepared for processing

Step 2: The average vector $\Psi$ is calculated

$$
\psi=\frac{1}{\mathrm{M}} \sum_{n=1}^{M} \Gamma_{n}
$$

Step 3: The difference between each face and the average is computed 


$$
\Phi_{i}=\Gamma_{i}-\psi
$$

Step 4: The empirical covariance matrix $C$ is calculated using the difference $\Phi$

$$
C=\frac{1}{M} \sum_{n-1}^{M} \Phi_{n} \Phi_{n}{ }^{T}
$$

Step 5: The eigenvectors (eigenfaces) $u_{i}$ and corresponding eigenvalues $\lambda_{i}$ of the covariance matrix are calculated and the eigenvectors normalized.

Step 6: Eigenvectors with high eigenvalues are selected from the $M$ eigenvectors (eigenfaces) $u_{i}$ based on the principal component analysis which is to find the vectors that best represent the distribution of the face images in the training set. Generally the features of the face are better described by the eigenvectors with higher eigenvalues.

The testing phase involves computing the weights of an image to be matched by projecting it onto the space of the eigenfaces extracted from the training set and comparing with images in the database.

$$
\begin{aligned}
& \omega_{k}=u_{k}^{T}\left(\Gamma_{\text {new }}-\Psi\right), \quad k=1 \ldots . . M \\
& \text { and form vectorof weights } \\
& \Omega_{\text {new }}{ }^{T}=\left[\omega_{1} \omega_{2} \ldots \ldots . \omega_{M^{\prime}}\right]
\end{aligned}
$$

with $M^{\prime}<M$, and

$\omega_{k}$ being weights of query image,

and, $\Omega_{\text {new }}$ being the corresponding vector of the weights.

The Euclidean distance between the weights of the query image and each image in the database is computed as $d\left(\Omega_{i}, \Omega_{i}\right)$ which provides a measure of similarity between the corresponding images $i$ and $j$. 
The Eigenphase algorithm [24] is somewhat similar to the Eigenface algorithm discussed above. The main difference being the domain in which the principal component analysis (PCA) is performed. For the Eigenface algorithm, PCA is performed in the space domain and for the Eigenphase algorithm, PCA is performed in the frequency domain.

The Eigenphase algorithm does not make use of the magnitude spectrum; PCA is performed in the frequency domain on only the phase spectrum [39] of the face images.
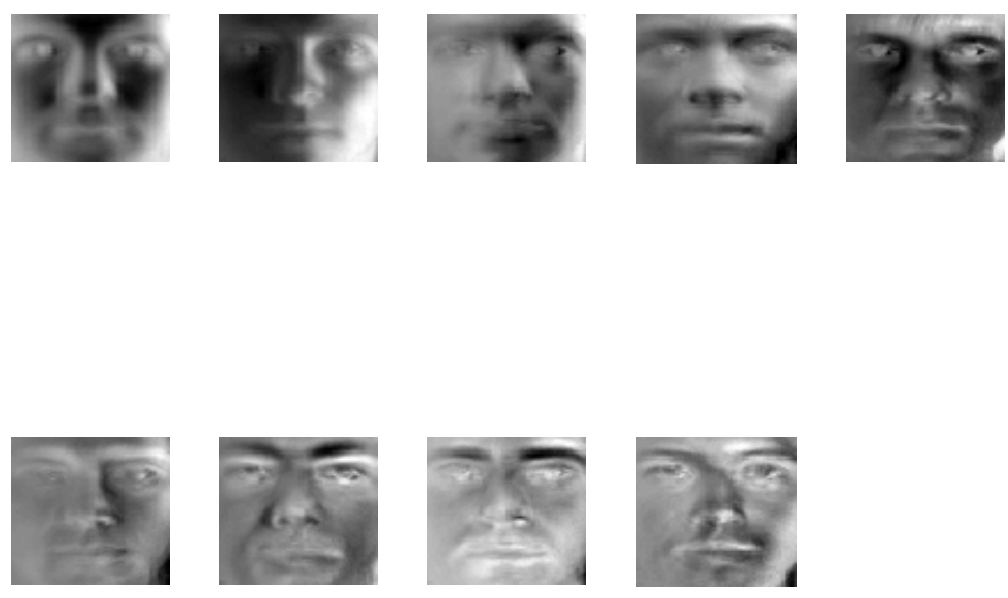

Figure 2.1: A set of Eigenfaces

\section{Euclidean Distance}

The Euclidean distance measures the similarity between two images. If the computed distance is below a pre defined threshold value, the two faces compared is concluded as a match; on the other hand if the computed distance surpass the threshold value the images are not a match. 
Given two n dimensional vectors $x$ and $y$ such that

$$
\begin{aligned}
& x=\left[x_{1}, x_{2}, x_{3}, \ldots . . . ., x_{n}\right], \text { and } \\
& y=\left[y_{1}, y_{2}, y_{3}, \ldots \ldots . . . ., y_{n}\right] .
\end{aligned}
$$

The Euclidean distance is computed as

$$
d(x, y)=\sqrt{\sum_{i=1}^{n}\left(x_{i}-y_{i}\right)^{2}} .
$$

\section{Eigenvectors and Eigenvalues}

An eigenvector of a matrix is a vector such that, if multiplied with the matrix, the result is always an integer multiple of that vector. This integer value is the corresponding eigenvalue of the eigenvector. This relationship can be described by the equation $e \times u=\lambda \times u$, where $u$ is an eigenvector of the matrix $e$ and $\lambda$ is the corresponding eigenvalue.

Eigenvectors possess the following properties [3]:

- $\quad$ They can be determined only for square matrices

- $\quad$ There are $n$ eigenvectors (and corresponding eigenvalues) in an $n \times n$ matrix.

- All eigenvectors are orthogonal, i.e. at right angle with each other. 


\subsection{Defocus Blur}

Defocus blur [4] is a type of noise in an image and is generally caused by camera focusing error. The degree of defocus increases as the distance between an object and the focal point increases. Practically defocus blur can be caused by zooming in and out. Figure 2.2 shows two images of different blur strengths. Synthetic defocus blur can be simulated by filtering the image with an averaging filter.
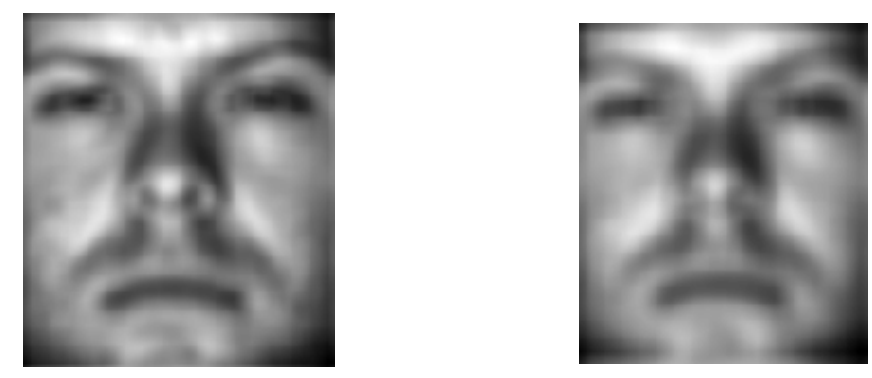

Figure 2.2: Defocused images at varying strengths; left (10), right (15).

\subsubsection{Averaging Filter}

An averaging filter is a low pass filter. In general, low pass filters are used to reduce the effect of noise in an image, but they can also be used to blur an image. The degree of blur increases as the filter size increases.

The average filter works by computing the average of the gray-level values within a filter window surrounding each pixel. The pixel values in the filter window is summed up and divided by the number of pixels in the window. Each pixel value is replaced by the corresponding average. Figure 2.3 demonstrates the operation of the averaging filters. 


$\left.\begin{array}{llll}a 11 & a 12 & a 13 & a 14 \\ a 21 & a 22 & a 23 & a 24 \\ a 31 & a 32 & a 33 & a 34 \\ a 41 & a 42 & a 43 & a 44\end{array}\right] \Rightarrow 2 \times 2$ Averaging Filter $\Rightarrow \begin{array}{llll}a 11^{\prime} & a 12^{\prime} & a 13^{\prime} & a 144^{\prime} \\ a 21^{\prime} & a 22^{\prime} & a 23^{\prime} & a 24^{\prime} \\ a 31^{\prime} & a 32^{\prime} & a 33^{\prime} & a 34^{\prime} \\ a 41^{\prime} & a 42^{\prime} & a 43^{\prime} & a 444^{\prime}\end{array}$

$$
\begin{aligned}
& a 11^{\prime}=\frac{a 11+a 12+a 21+a 22}{4} \\
& a 14^{\prime}=\frac{a 14+a 24+a 11+a 21}{4}
\end{aligned}
$$
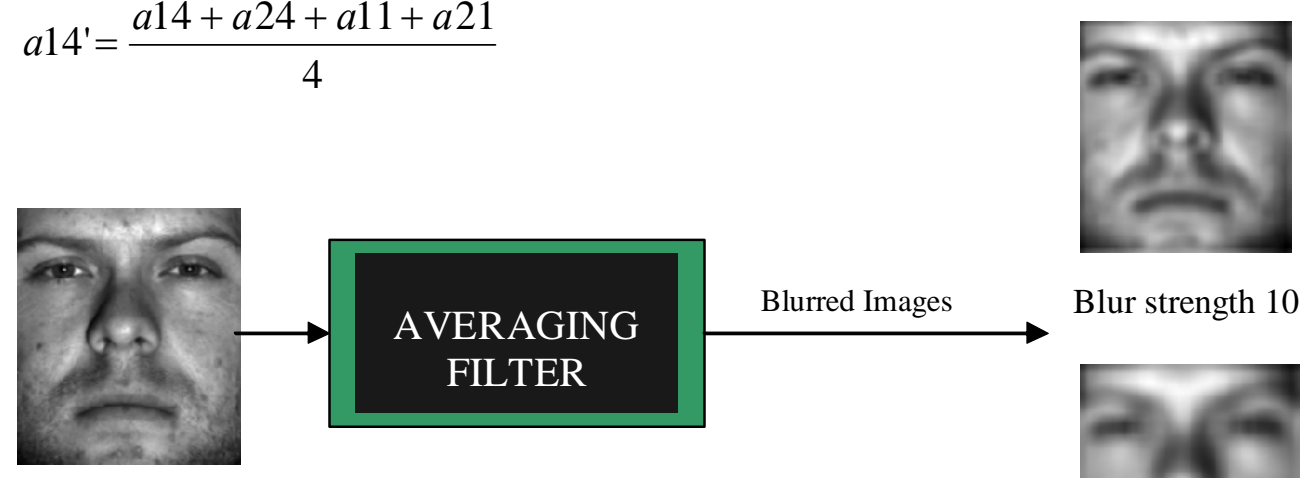

Blur strength 10

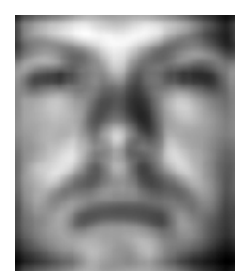

Blur strength 15

Figure 2.3: Averaging Filter

\subsection{Motion Blur}

Motion blur [19] [27] [29] is type of image distortion caused by a relative motion between a camera and the object (target) during exposure time. In some applications, motion blur is desired; used for visual effects and other purposes. In facial recognition, motion blur is an undesired effect. It degrades the quality of the image by changing the features of the image which in turn degrades the performance of face recognition algorithms. Motion blur is characterized by two parameters - the direction of the blur and the strength of the blur. In 
terms of these variables, linear motion blur is defined as the shift of the pixels in an image by "x" pixels at a "theta" degree angle, were $\mathrm{x}$ is the strength and theta, the direction. Motion blur is modeled as:

$$
I(x, y)=f(x, y) \times h(x, y),
$$

where $f(x, y)$ is the original non blurred image, $h(x, y)$ the point spread function causing the blur and $I(x, y)$ is the blurred image. Majority of the existing methods used to estimate motion blur assumes that the point spread function is known, which is not always the case. The method used in this thesis makes no a prior assumptions. Motion blur is synthetically simulated by convolving the original image with a linear filter. Figure 2.4 displays synthetically motion blurred images

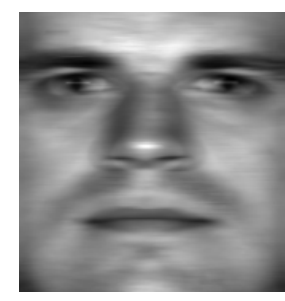

a

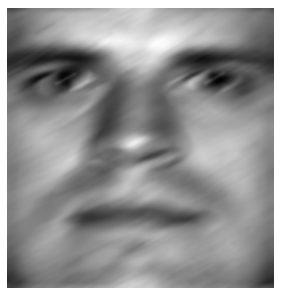

b

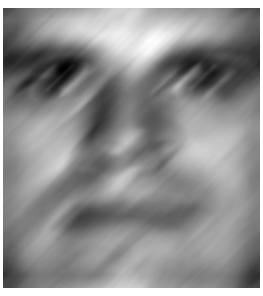

C

Figure 2.4: Motion blurred images at varying strengths and direction. (a) strength 15 @ $0^{0}$ angle, (b) strength15 @ 30 angle, (c) strength 25 @ 45 angle
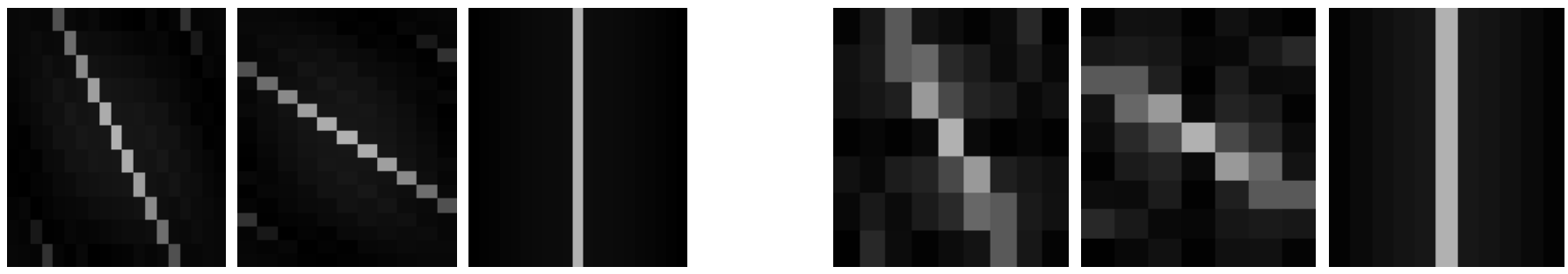

Fig 2.5: Fourier transform of filter used to simulate synthetic motion blur at varying strengths and orientation, left to right $\left(\sim 30^{0}, \sim 60^{\circ}, \sim 0^{0}\right)$ 


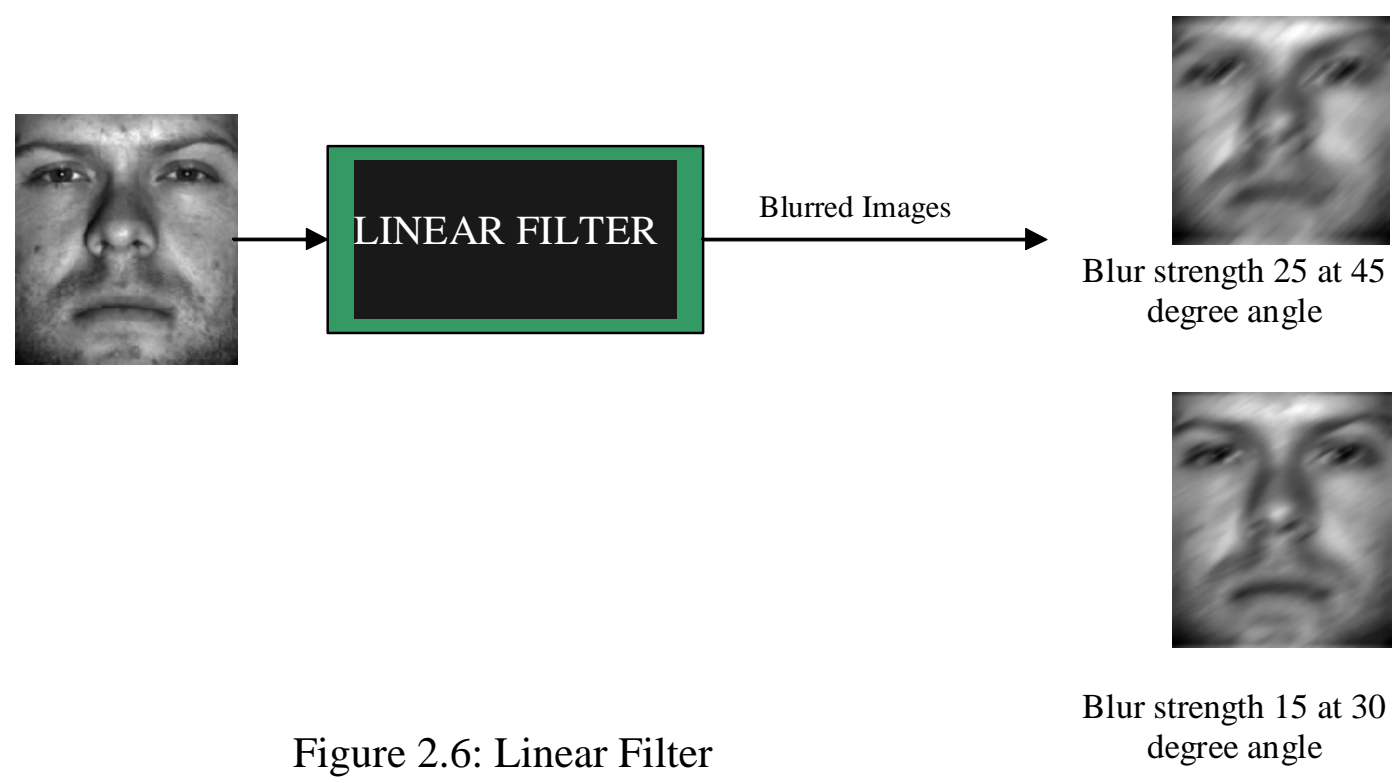

Figure 2.5 shows the fourier transform of the filter used to simulate synthetic motion, while Figure 2.6 demonstrates the operation of this filter.

\subsection{Lighting}

External lighting is often used when capturing face images. This external lighting can cause images to be degraded depending on the exposure.

A captured image would be lit evenly or unevenly depending on the lighting environment (condition) during exposure. The brightness and contrast of an image would vary with respect to this lighting condition. An overexposed or underexposed face images resulting from inappropriate light exposure, results in an uneven brightness and poor image contrast. While the human eye may be able to accurately recognize images captured under different lighting conditions, most existing facial recognition systems are not capable of accurately matching or recognizing these images [35]. Uneven lighting may also be 
attributed to the effects of the spectral characteristics of the sensor used [15] as well as the users knowledge and operation of the capturing device. The Yale database provides images of different lighting conditions used in this thesis to conduct our performance study (see figure 2.7, the top row). For the WVU database, we simulated brightness by increasing the amplitude of the image pixels. This is done by simply multiplying the image matrix by a numerical value. Multiplying the image matrix by a number greater than 1 will increase the brightness and multiplying by a number less than one will darken the image (see examples in figure 2.7, the bottom row).
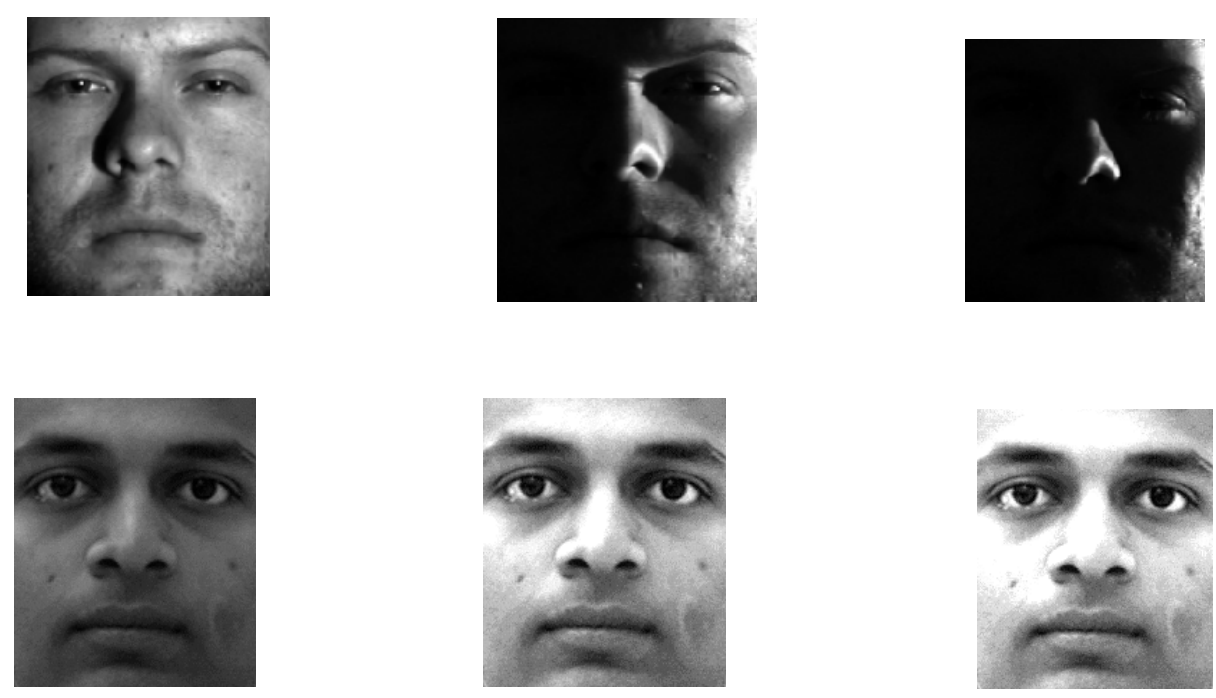

Figure 2.7: Lighting at varying conditions; top row (contrast), bottom row (brightness)

To further study the effect of Lighting on recognition we enhanced the degraded images by performing histogram equalization and conducted the performance test again. Results will be presented in chapter 3. 


\subsection{Occlusion}

Facial occlusion could be described as an obstruction of any of the features of the face. A common facial occlusion occurs when the individual hair covers a significant portion of the face. Occlusion reduces the amount of information available which reduces the amount of useful features that can be extracted and ultimately degrade recognition performance. We simulated occlusion by masking out portions of the face (see examples of modified images in figure 2.8).
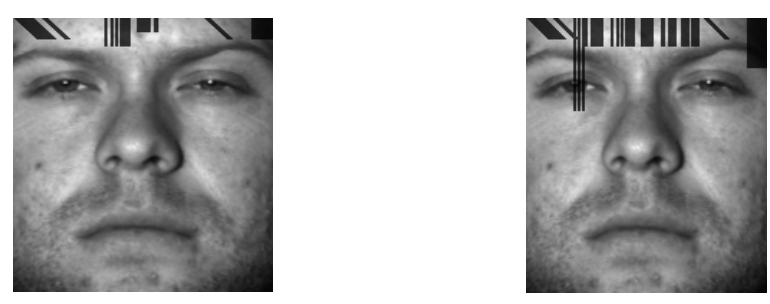

Figure 2.8: Synthetically occluded images

\subsection{Cropping}

Cropping is simulated by deleting the outside rows and columns of the image matrix. Increasing the number of rows and columns deleted, increases the level of cropping . Excessive cropping reduces the amount of information (features) that can be extracted from the face for recognition. Research has shown that the more the features contained in the face and available for recognition, the better the performance (figure 2.9 shows examples of cropped images). 

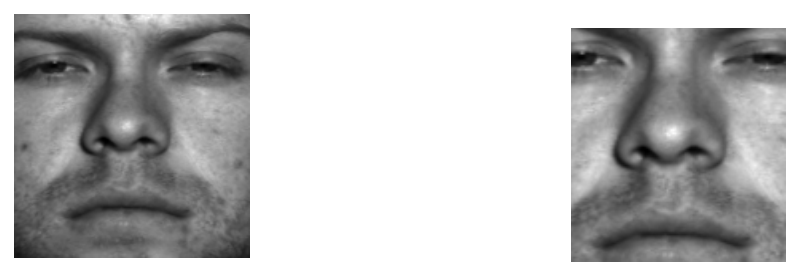

Figure 2.9: Cropped images

\subsection{Pixel Count}

Pixel count is the resolution of the image attributed to the resolution of the capturing device. Variation in pixel count may be attributed to the relative distance between the subject and the camera or device used in capturing the face image. We synthetically simulate pixel count by averaging windows of pixels and replacing that window with the resulting average (figure 2.10 displays face images of different resolutions).
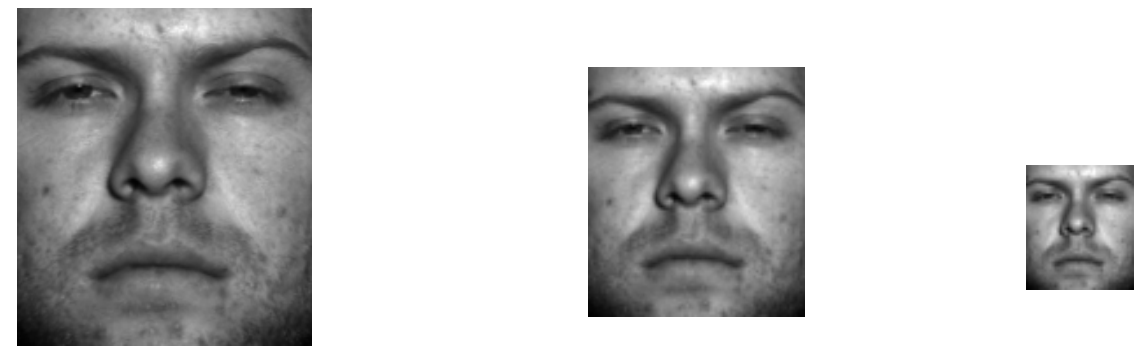

Figure 2.10: Face Images at different resolution (a) image size $192 \times 168$, (b) image size $96 \times 84$, (c) image size $48 \times 42$ 


\subsection{Facial Expression}

Facial Expression is also a factor researched to affect performance. The Yale database provides images of a user with varying facial expressions including but not limited to smiling, sad and happy. We did not have to simulate expression.
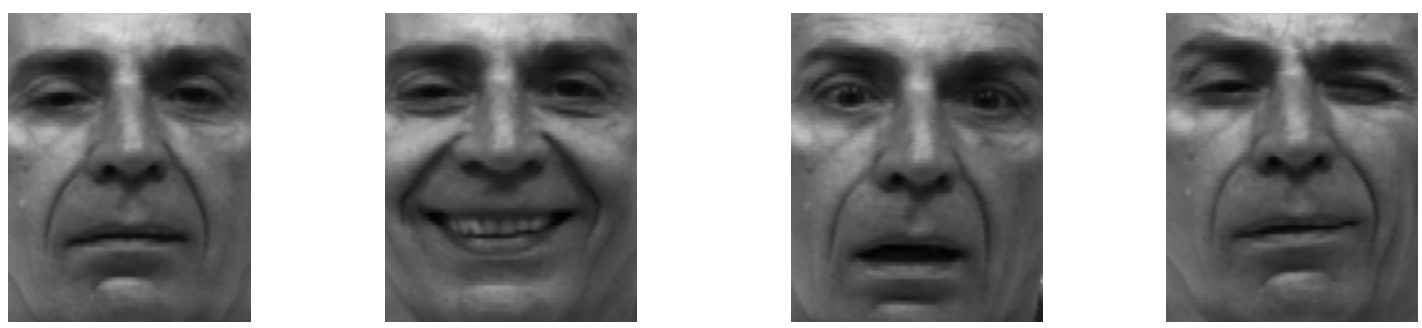

Figure 2.11: Different Facial Expressions. From left to Right (Normal, Smiling, Surprised and Winking) 


\section{Chapter 3}

\section{Synthetic Studies}

All experiments follow the model depicted in Figure 3.1 below. A template is generated for each user from the combination of the eigenfaces [3] [24] [25] extracted from the training set. The test images are projected onto the space of the extracted eigenfaces to compute the weights which are compared to those of the images in the database to generate the performance score.

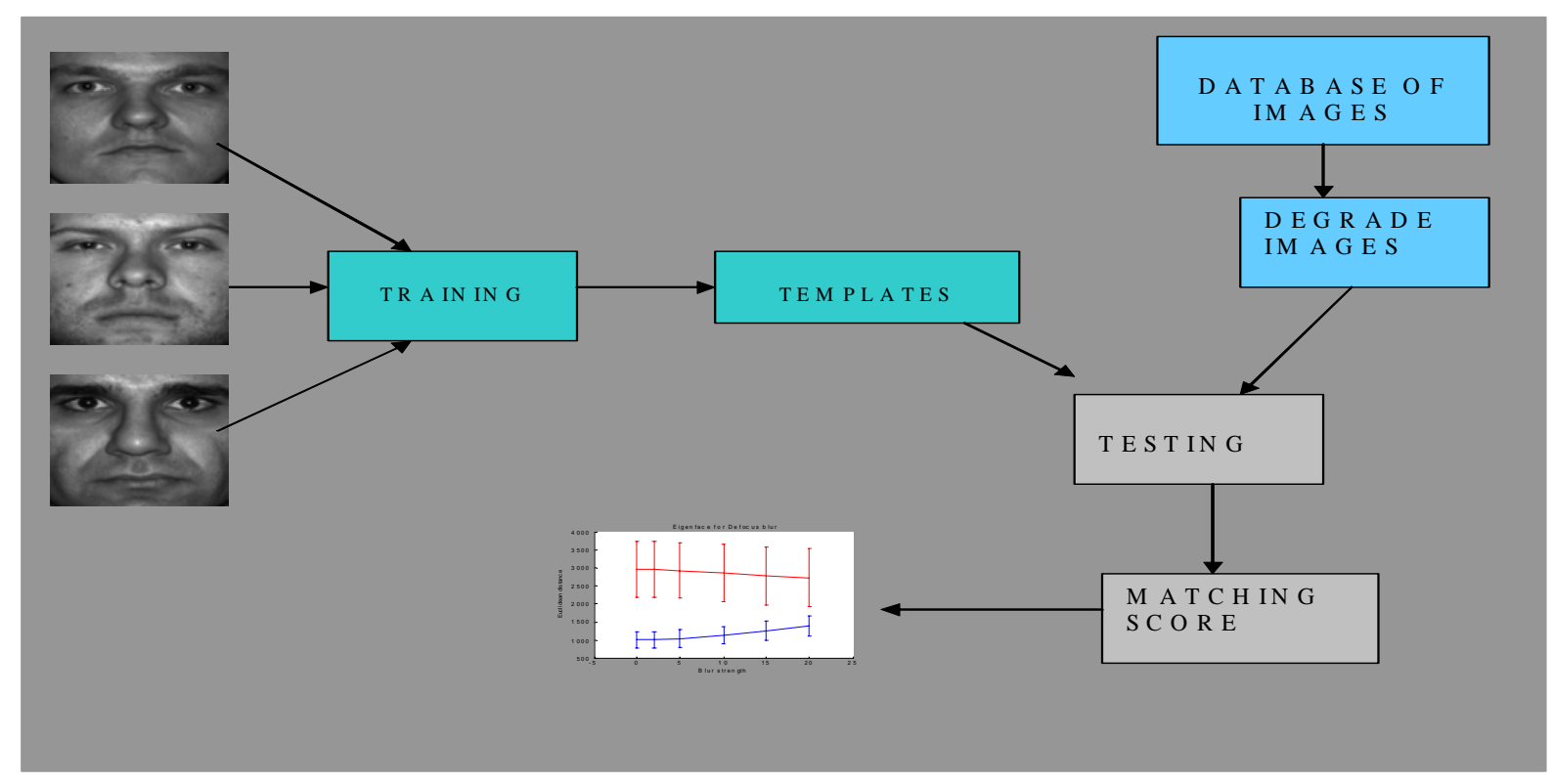

Figure 3.1: Model for Recognition Performance study 


\subsection{Yale Database}

Effects of Defocus blur, motion blur, lighting, cropping, pixel count, occlusion, and expression on recognition performance were studied using the Yale database. A total of 39 images from the Yale database were trained using the eigenface [3] [24] [25] and eigenphase [24] algorithm (one visually good image from each user), while 5 (or 6) levels of degradation was simulated for each quality factor and used for testing. We generate and plot the euclidean distance for the imposter and genuine score. The results are shown in Figure 3.2 through Figure 3.8. The blue lines represent the genuine score, while the red lines represent the imposter scores.

\section{Defocus Blur}
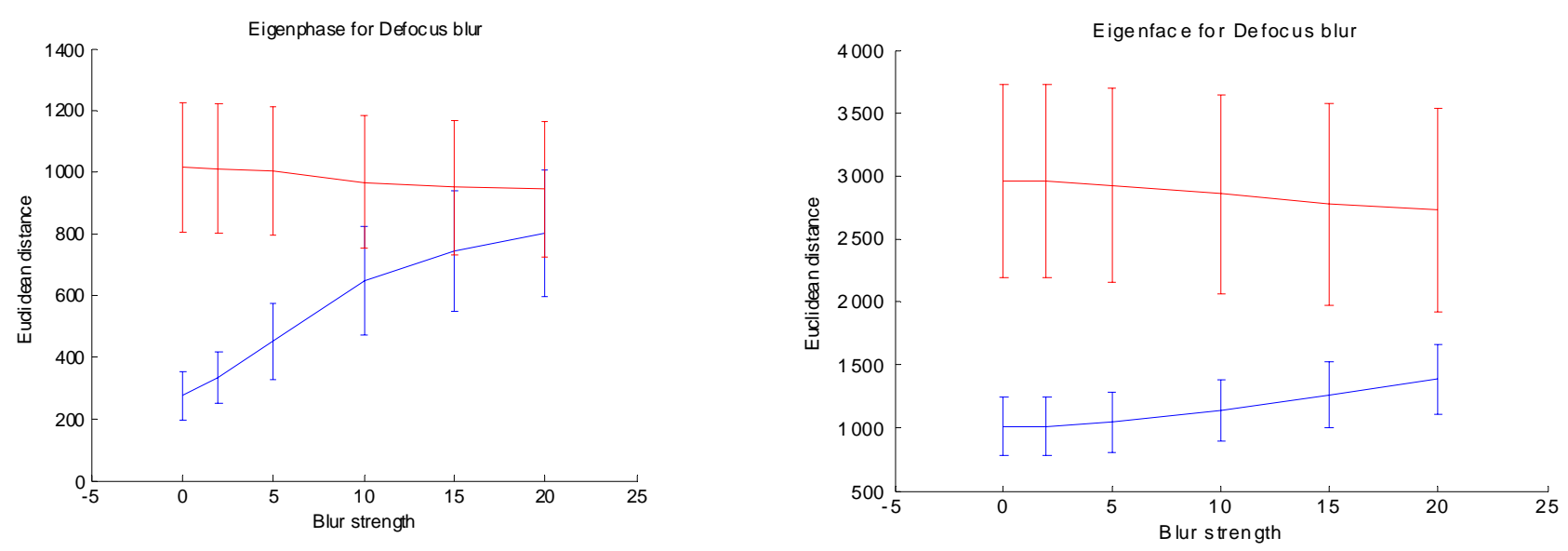

Figure 3.2: Matching score for defocus blur. Eigenphase (left), Eigenface (right) 


\section{Motion Blur}
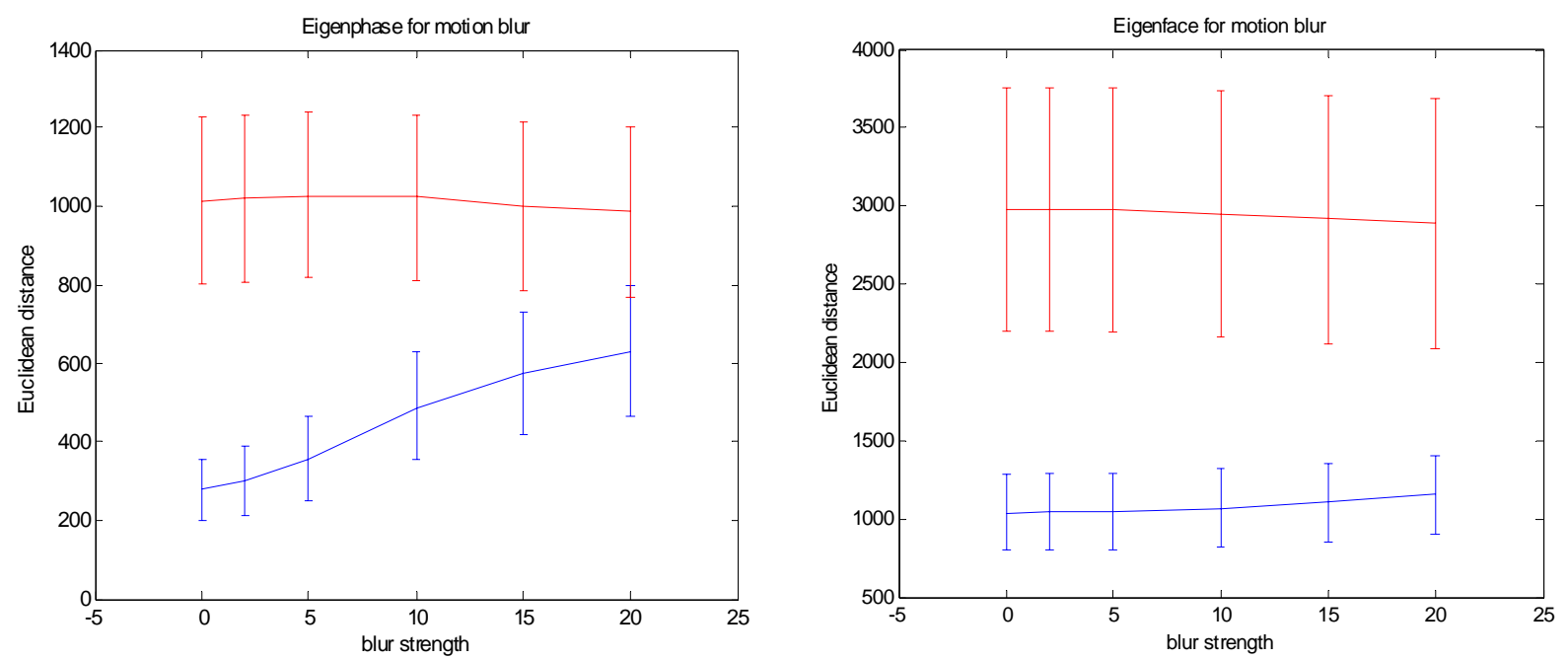

Figure 3.3: Matching score for motion blur. Eigenphase (left), Eigenface (right)

\section{Lighting}

\section{Before Histogram Equalization}
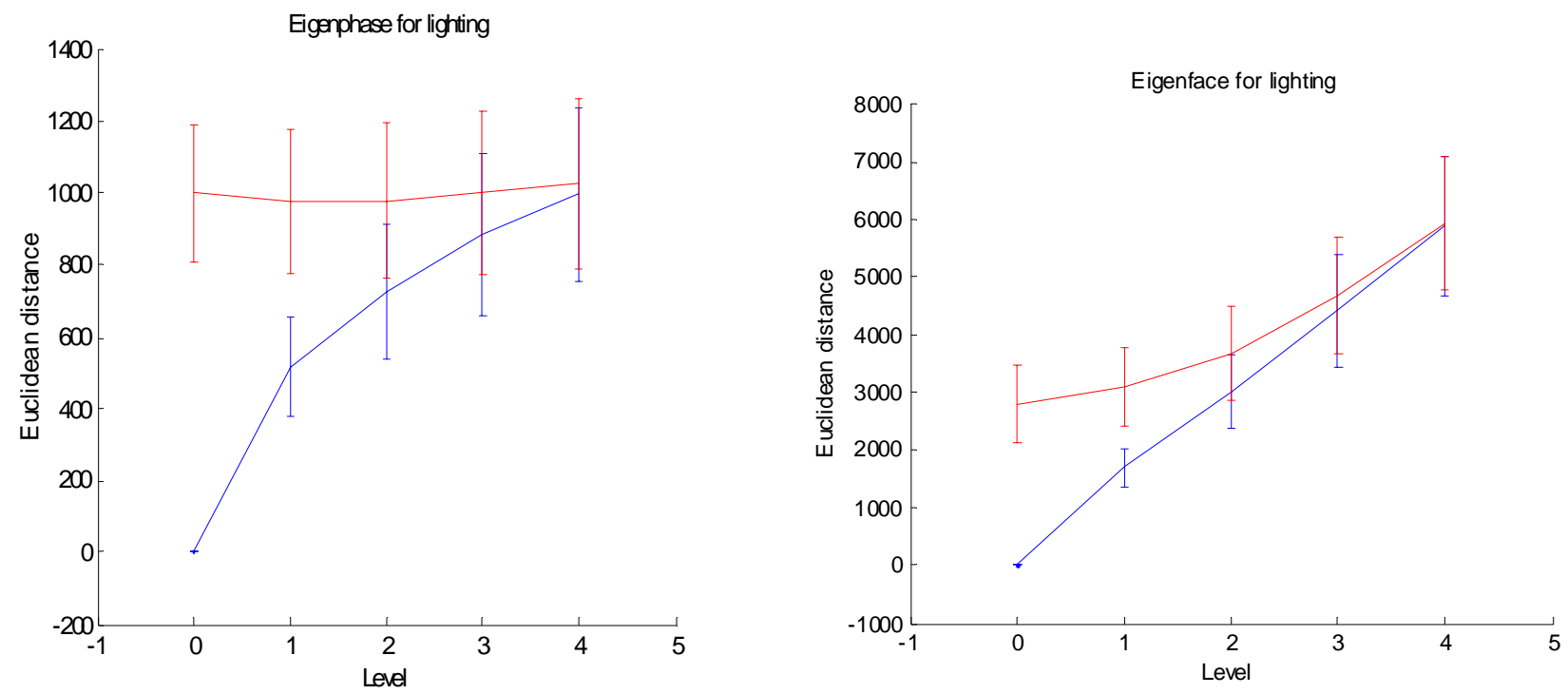


\section{After Histogram Equalization}
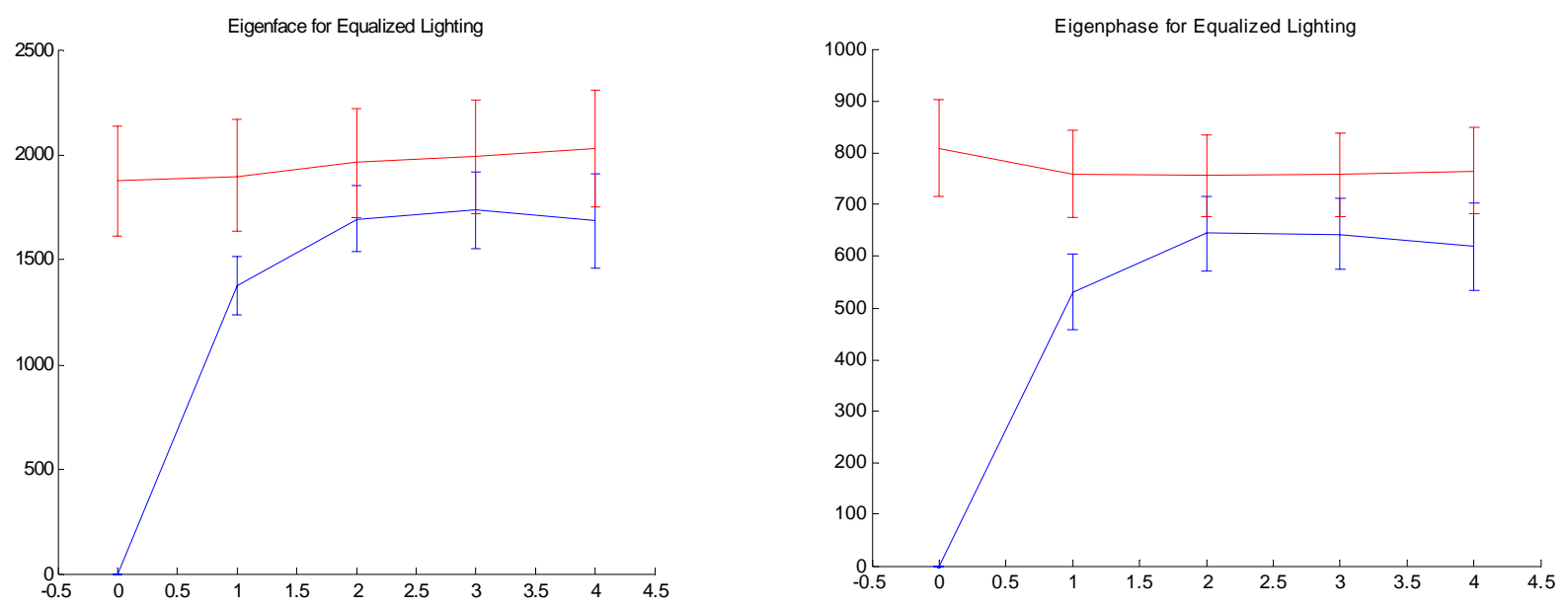

Figure 3.5: Matching score for Lighting. Eigenphase and Eigenface for lighting before histogram equalization (Top right and left respectively); Eigenface and Eigenphase for lighting after histogram equalization (Bottom right and left

\section{Cropping}
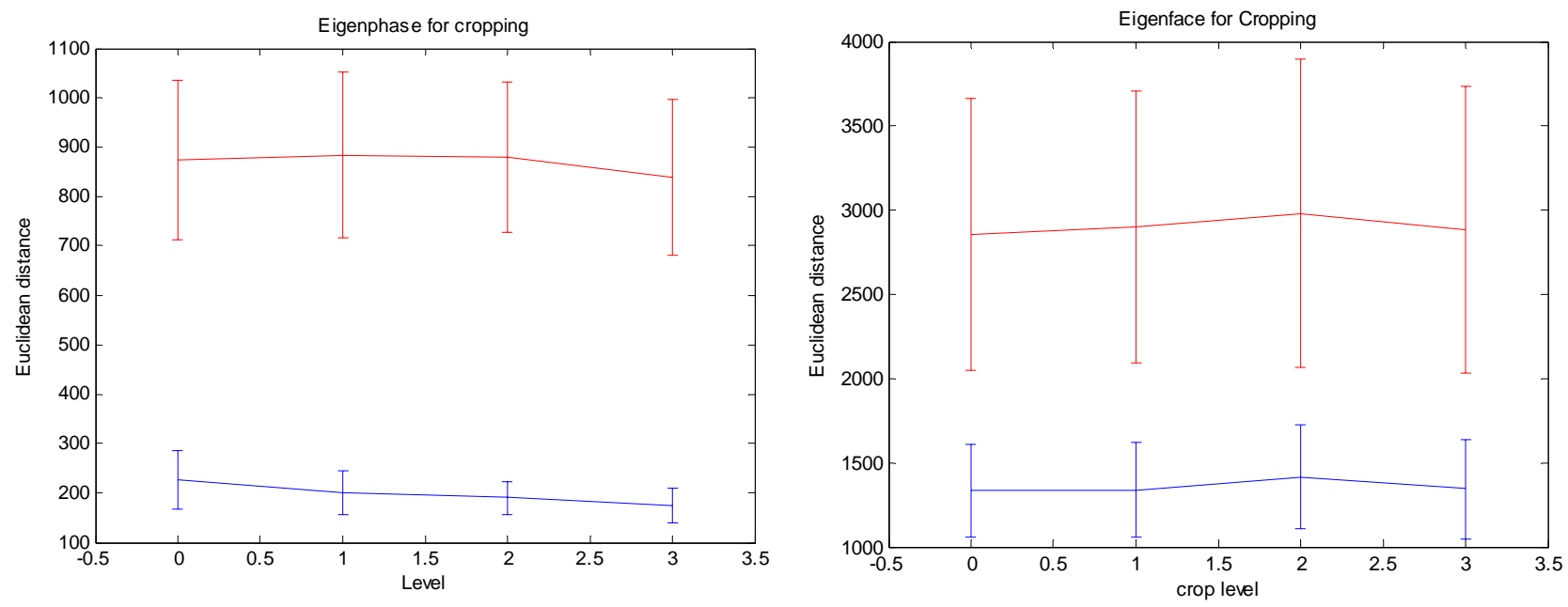

Figure 3.5: Matching score for cropping. Eigenphase (left), Eigenface (right) 


\section{Pixel Count}
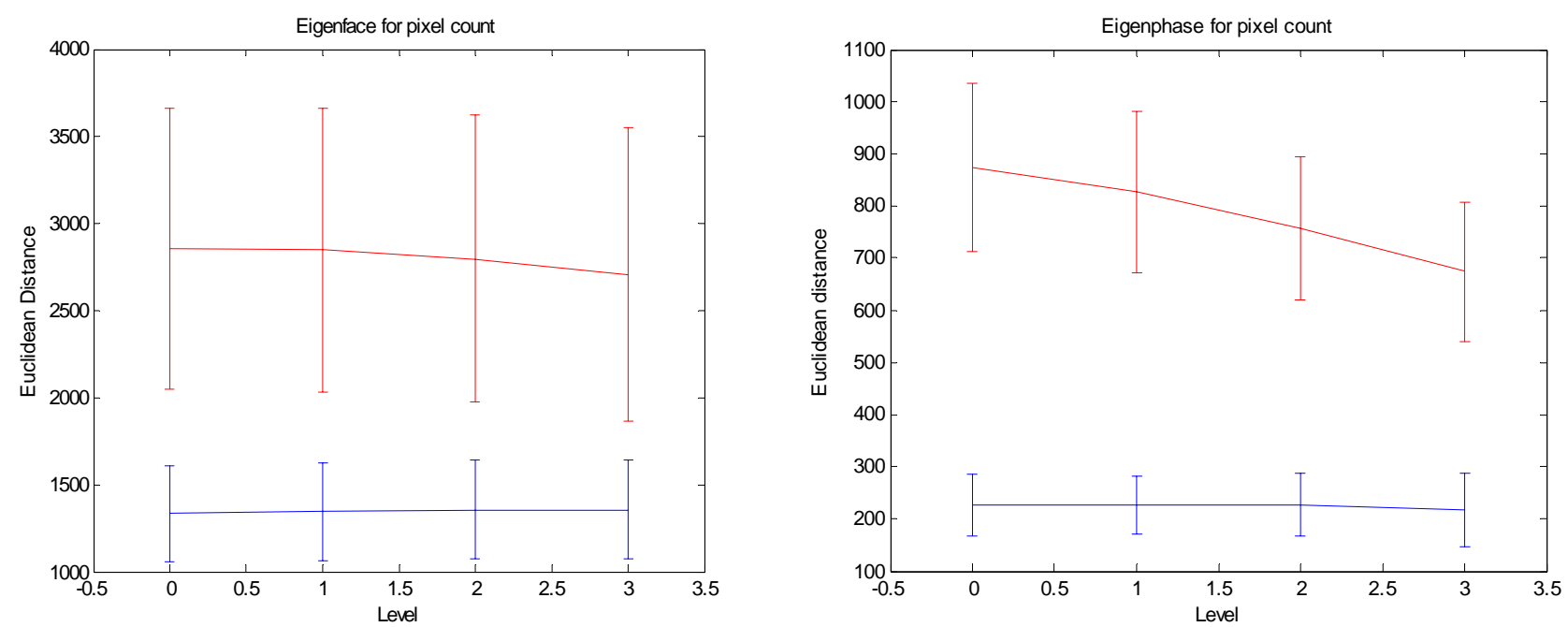

Figure 3.6: Matching score for Pixel Count. Eigenphase (left), Eigenface (right)

\section{Occlusion}
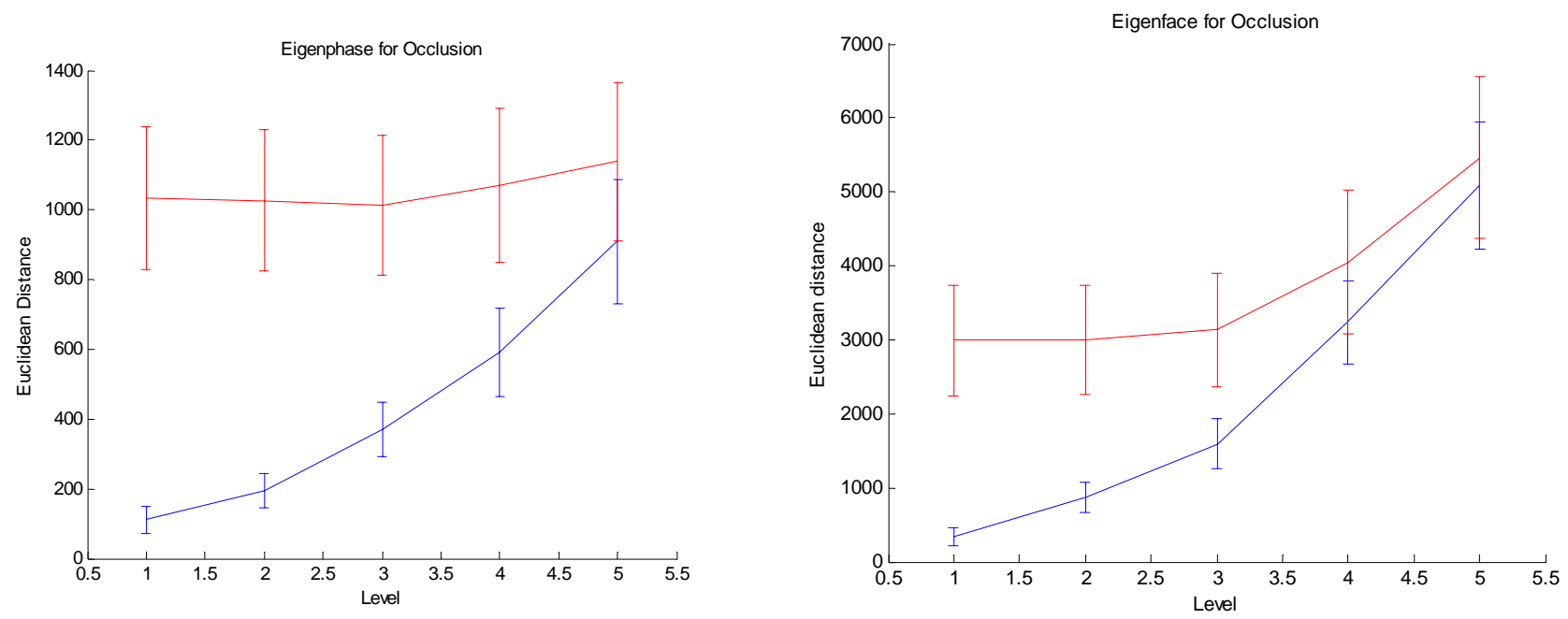

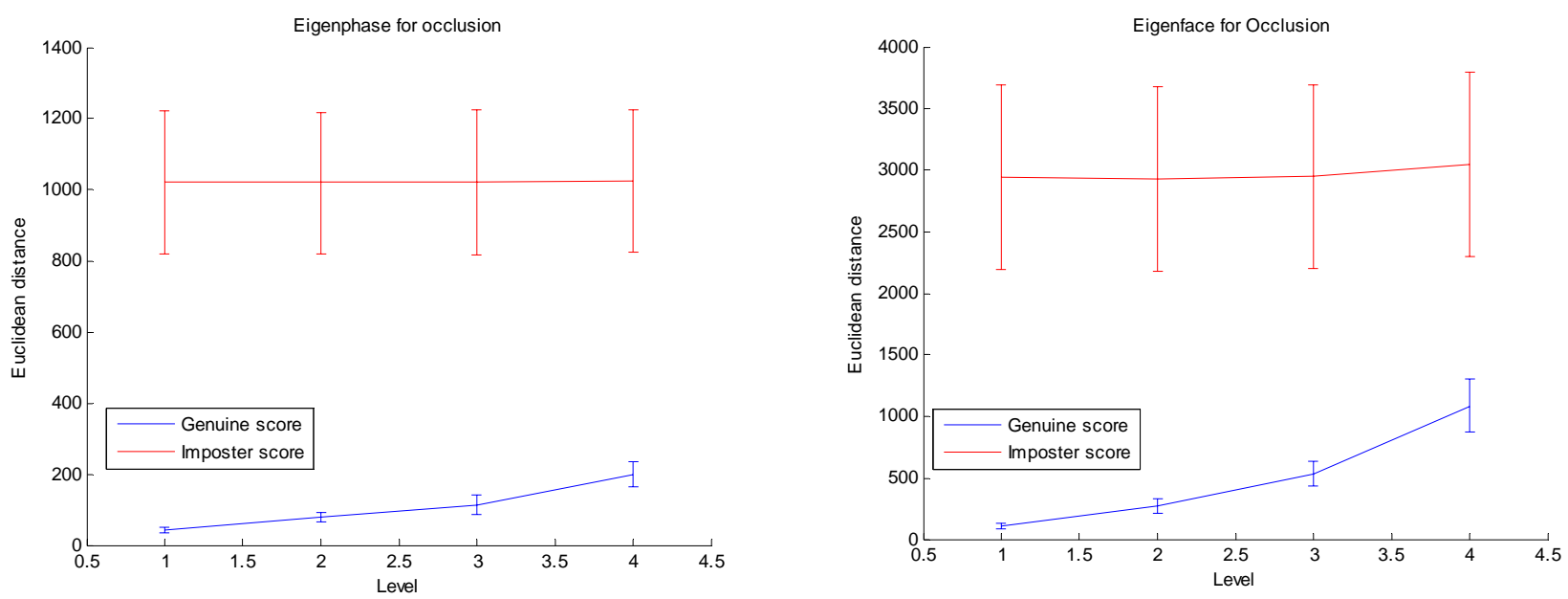

Figure 3.7: Top (matching score for Occlusion), Bottom (matching score for smaller Occlusion). Eigenphase (left), Eigenface (right).

\section{Expression}

The horizontal axis in Figure 3.8 is represented as follows:

$1=$ Center Light

$2=$ Glasses

3 = Happy

$4=$ Left Light

$5=$ No glasses

$6=$ Normal

7 = Right Light

$8=$ Sad

9 = Sleepy

$10=$ Surprised

$11=$ Wink

1,2,4,5 and 7 are not expression images, but rather are used for comparison. 

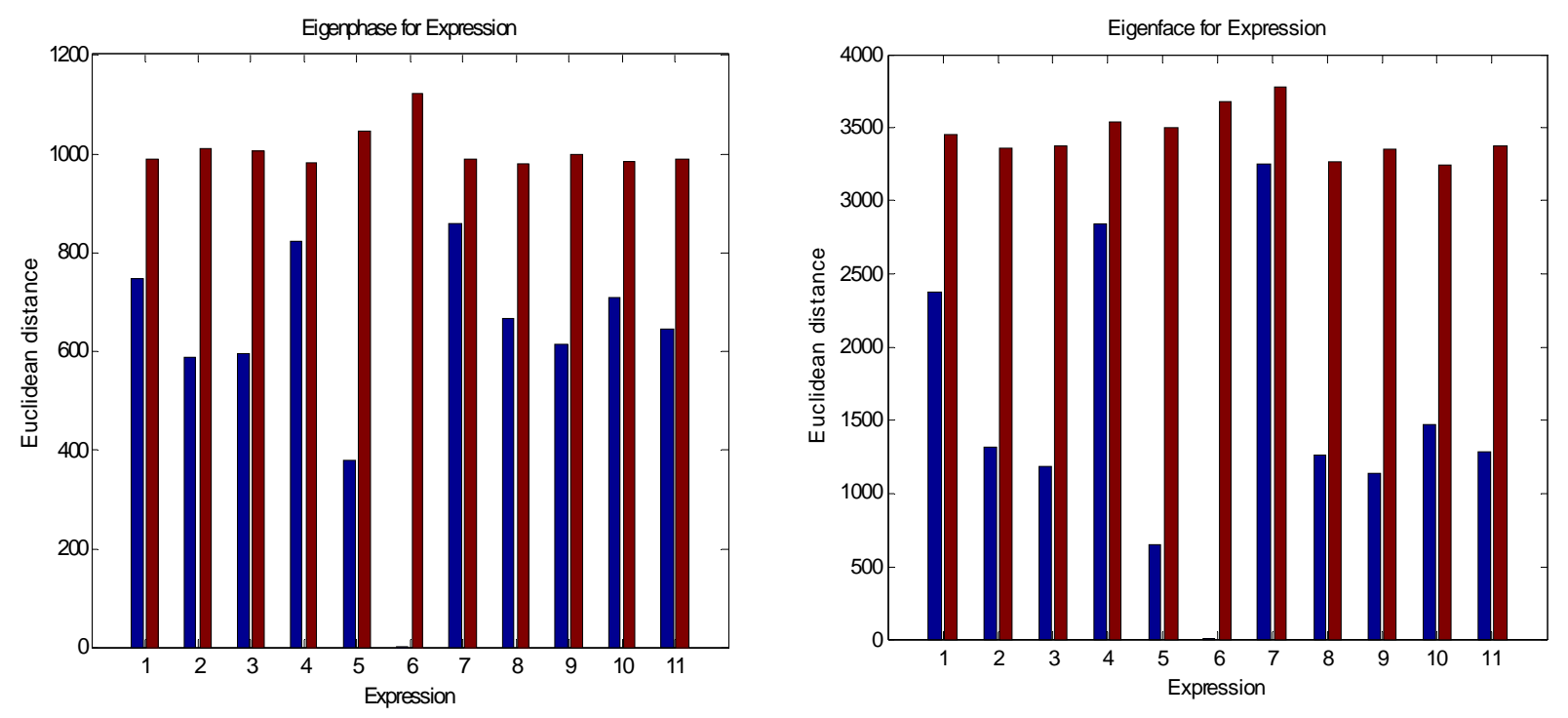

Figure 3.8: Matching score for Expression. Eigenphase (left), Eigenface (right)

\subsection{WVU Database Results}

Defocus blur, motion blur, lighting, pixel count, and occlusion were also studied using the WVU database. Similar to the Yale database, we trained a total of 30 images from the WVU database using the eigenface and eigenphase algorithm (one visually good image from each user), and simulated 5 (or 6) levels of degradation for each quality factor for testing. We generate and plot the euclidean distance for the imposter and genuine score. The results are shown in Figure 3.9 through Figure 3.13. The blue lines represent the genuine score, while the red lines represent the imposter scores. 


\section{Defocus Blur}
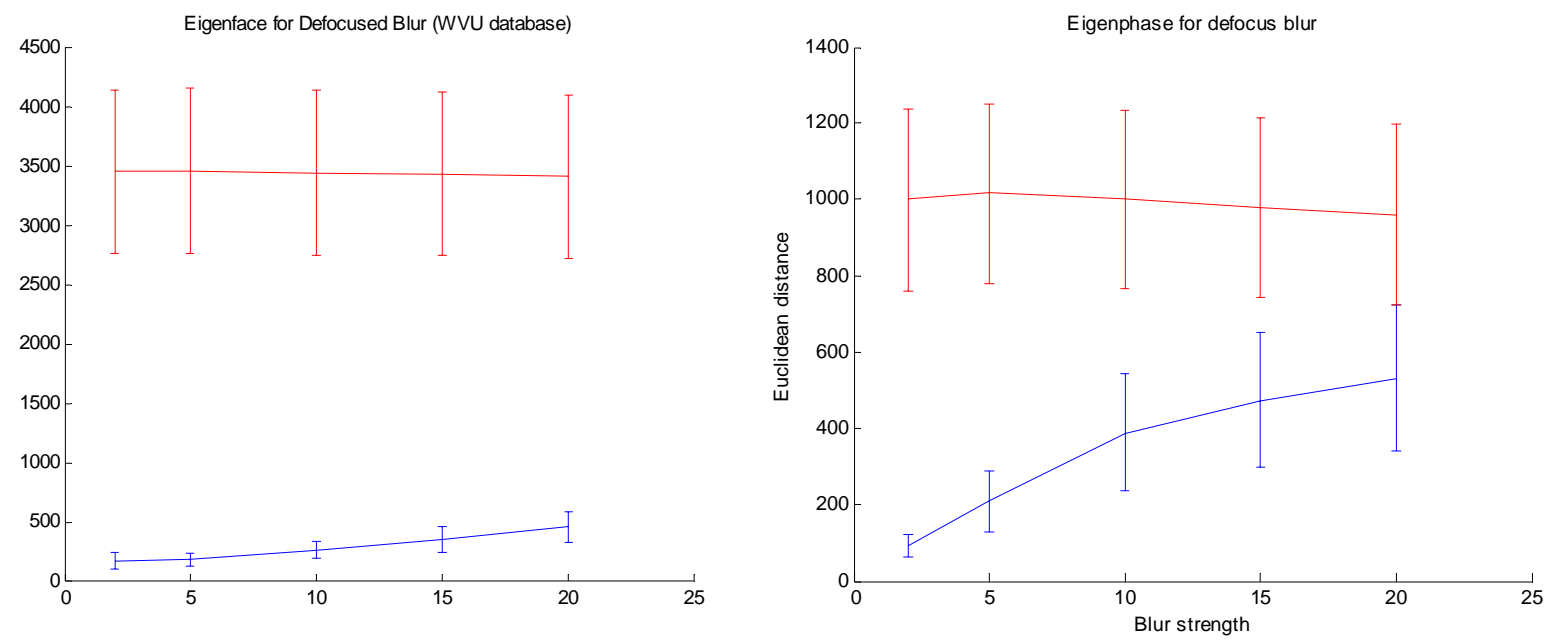

Figure 3.9: Matching score for defocus blur. Eigenface (left), Eigenphase (right)

\section{Motion Blur}
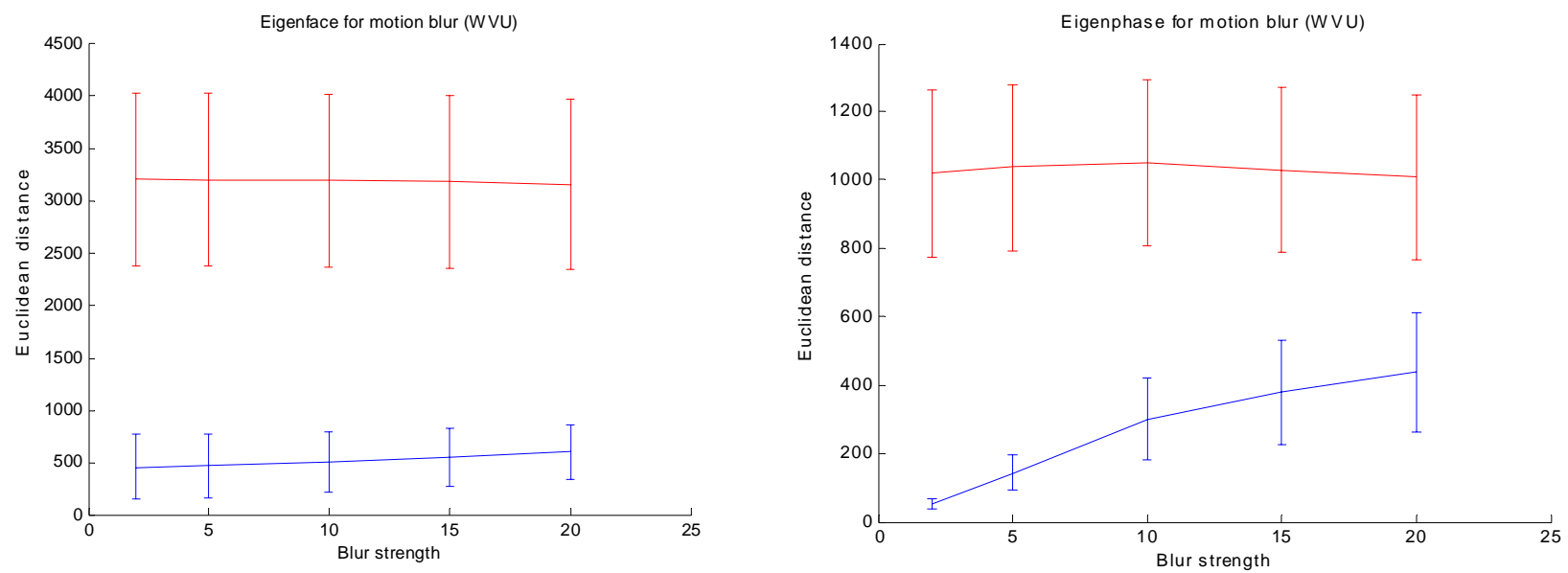

Figure 3.10: Matching score for Motion blur. Eigenface (left), Eigenphase (right) 


\section{Lighting (Brightness)}
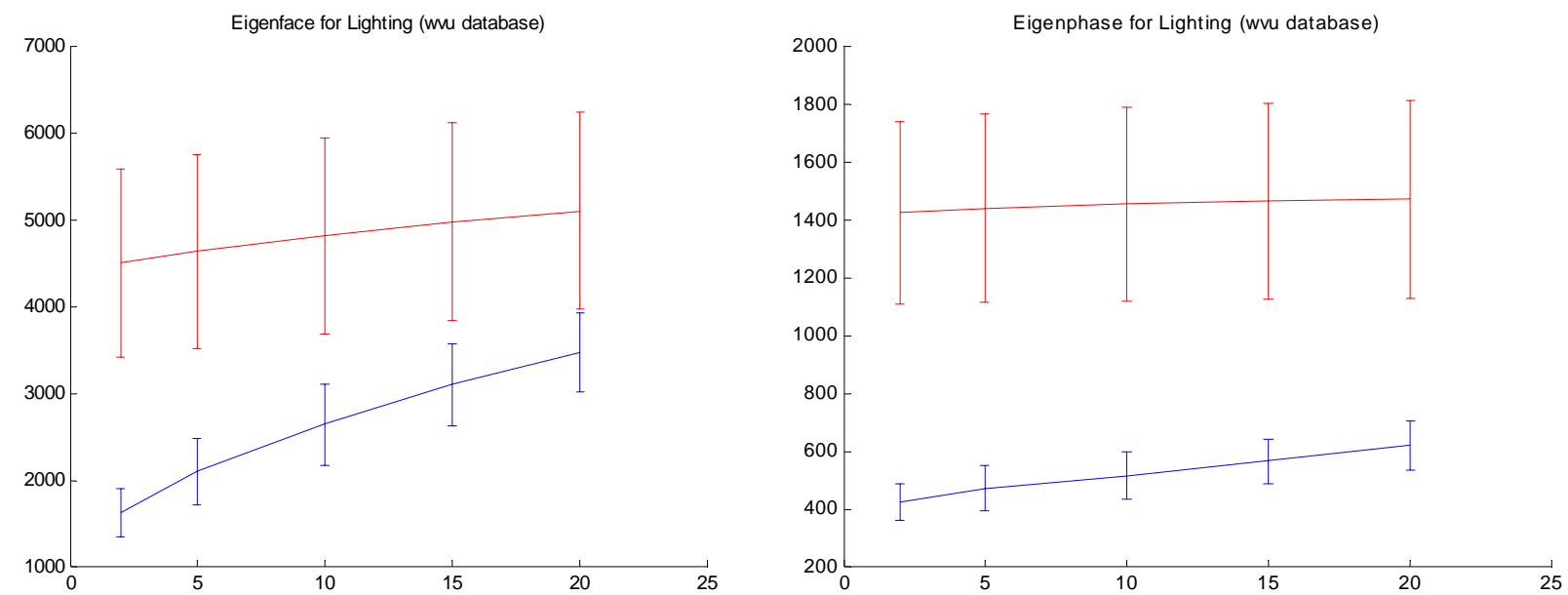

Figure 3.11: Matching score for Lighting. Eigenface (left), Eigenphase (right)

\section{Pixel Count}

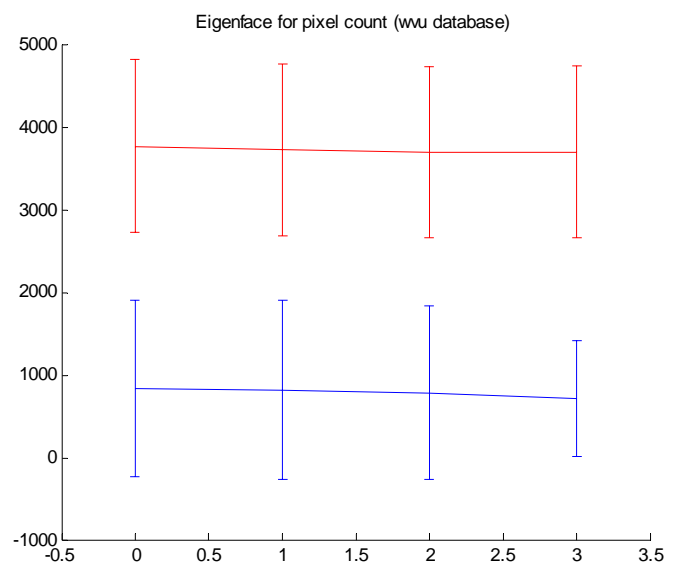

Eigenphase for pixel count (wvu database)

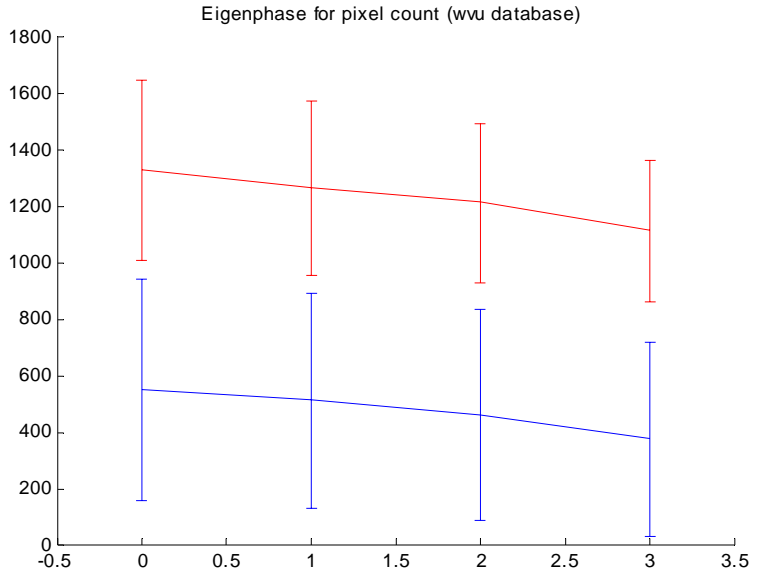

Figure 3.12: Matching score for Pixel Count. Eigenface (left), Eigenphase (right) 


\section{Occlusion}
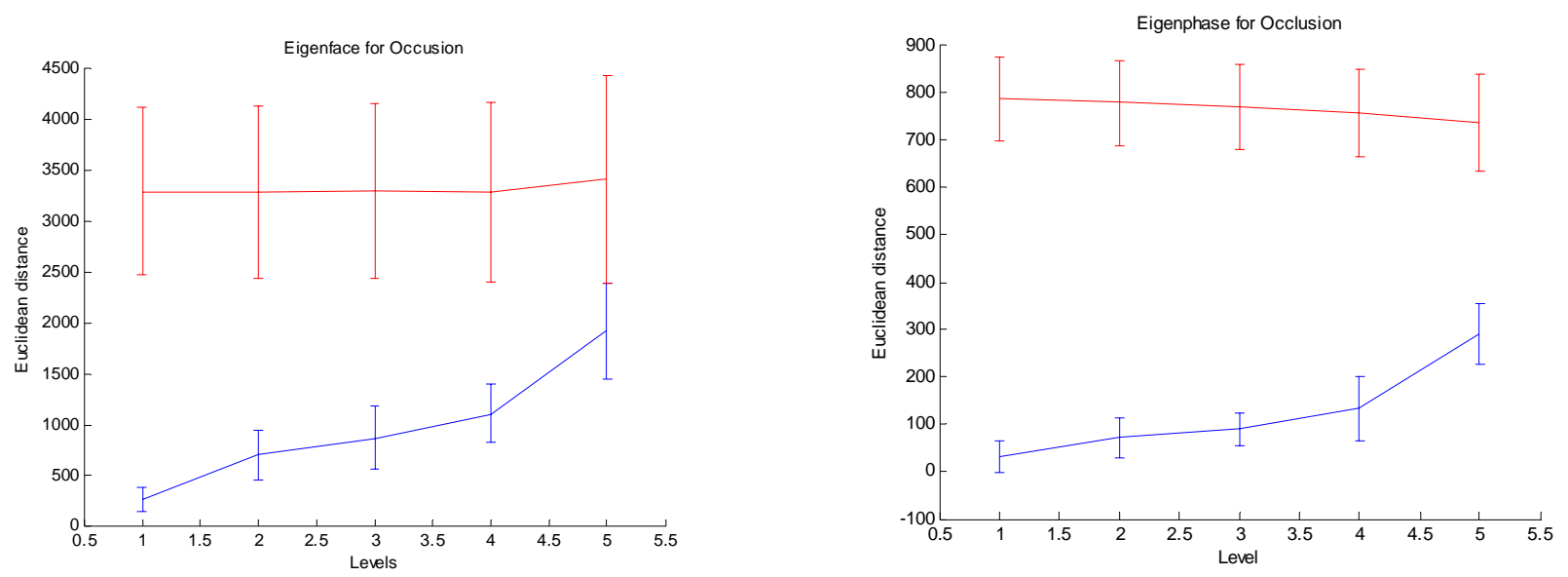

Figure 3.13: Matching score for Occlusion. Eigenface (left), Eigenphase (right)

Among all the face image quality factors studied, external lighting tends to affect facial recognition performance the most. It degrades the recognition performance of both the eigenface and eigenphase algorithms. As the variation among images increases and exposure (especially contrast) worsens, the separation between the genuine score and imposter score condenses (they approach each other). This separation increases the probability of matching error. Even though histogram equalization may not entirely restore the image, when applied, it separates the imposter score from the genuine score (se Figure 3.4) consequently improving performance.

Defocus blur and Motion blur seem to have a comparable effect on both algorithms studied. Both quality factors degrade the performance of the eigenphase algorithm. Similar to lighting, as the strength of blur increases, the separation between the imposter score and genuine score reduces (see Figure 3.2 and 3.3). For smaller blur strengths, the rate of degradation with a small increase in strength causes a sharp degradation in performance 
compared to that of a large blur strength; rate of degradation tends die down slowly for large blur strengths. The effects of defocus blur and motion blur on the eigenface recognition algorithm is minimal (see Fig 3.2 and 3.3). The degradation may be apparent at a high level blurs. Occlusion (see Figure 3.7) also tends to be a huge factor of recognition performance. The effect may not be as apparent at low occlusion levels (bottom row of Figure 3.7) but is quite degrading at high occlusion levels. Recognition performance on the other hand is robust to cropping and pixel count at small levels of degradation (see Figure 3.5 and 3.6 respectively). Unlike the other image quality factors, for expression we generate a bar plot. The blue bar represents the genuine score and the red bar represents the imposter score. To interpret the genuine and imposter scores, we plotted the scores for lighting variation and occlusion from glasses. These scores, along with that of a normal (non-degraded) image are used for comparison. We used the scores for lighting variation as the upper threshold and the score for the normal images as the lower threshold - From previous study of lighting variation, we know it greatly degrades performance recognition and hence expect a small difference between the average genuine score and average imposter score. For normal images we expect a much greater difference between the genuine and imposter score. If the difference between the genuine score and imposter score for a particular expression is closer to that of lighting variation we conclude that the variation in this expression degrades performance and on the other hand if the difference in scores is closer to that of the normal images, we conclude that the effect on recognition performance is minute. 


\section{Chapter 4}

\section{Quality Factor Estimation}

\subsection{Motion Blur}

Motion blur [19] [27] [29] as defined earlier is as result of the relative motion between the camera and the subject and is characterized by the direction of blur and strength of blur. Motion blur is modeled linearly as:

$$
I(x, y)=f(x, y) * h(x, y),
$$

where $f(x, y)$ is the original non blurred image, $h(x, y)$ the point spread function (PSF) causing the blur and $I(x, y)$ is the blurred image resulting from convolving $f$ and $h$.

As stated earlier most methods used to estimate motion blur assumes that the point spread function is known. In this thesis motion blur is estimated without any prior knowledge or assumption of the point spread function. Some of the other methods that have been proposed to estimate motion blur are

1. Use of the methods of moments which involves restoring the blurred image and comparing the moments of the resulting image to that of the original blurred image or searching for the best match of the image moments with the moments of images in a pre existing database [9]. 
2. Measuring the separation between zeros present in the PSF in the spectral domain $[10]$.

3. Use of maximum likelihood specifically for blurs with a symmetric PSF [36] [37].

The shortcomings of these methods are that assumptions are made that may not necessarily be valid in practice. PSF does not necessarily have zeros in the spectral domain and the shape or magnitude of the PSF is generally not known. Some of these methods also rely on an existing database of blurred images for training and classification to estimate the blur strength. The method discussed in this thesis estimates the blur direction and strength from a single blurred image and does not assume that the PSF or its shape is known. Neither does it rely on the presence of zeros in the image PSF in the spectral domain. The blur estimation method discussed in this thesis is broken down into two stages -

Stage 1: Estimation of the direction of the blur smear and

Stage 2: Estimation of the strength of the blur.

The estimation of the direction of blur makes use of the properties of the Fourier transform. The next section gives a brief description of the Fourier Transform. 


\section{Fourier Transform and its properties}

Fourier transform (FT) transforms a function from the spatial domain to the frequency domain. Inverse Fourier transform does just the opposite; it transforms a function from the frequency domain to the spatial domain. Fourier transform could either be continuous or discrete. This thesis makes use of the discrete Fourier transform. Equation 4-2 gives the mathematical representation of the 2d continuous Fourier transform [38].

$$
\left.F\{f(x, y)\}=f\left(x^{\prime}, y^{\prime}\right)=\int_{-\infty}^{\infty} \int_{-\infty}^{\infty} f(x, y) e e^{-j 2 \pi\left(x x^{\prime}+y y^{\prime}\right)}\right] d x d y
$$

The square of the magnitude of the Fourier transform is referred to as the power spectrum. The features of the Fourier transform are amplified by taking the logarithm of the Fourier transform of the image which pronounces little details in the image and makes it more visible.

A very important property of the Fourier transform used in this thesis is the convolution theorem. This theorem states that, the Fourier transform of a convolution of two functions is equal to the product of the Fourier transform of the two functions. In lieu of this, convolution in the spatial domain can be computed by simply multiplying the Fourier transforms in the frequency domain [38].

\subsubsection{Estimation of the direction of blur}

In order to estimate the direction of the motion smear, the directional properties of the Frequency spectrum of the blurred image are examined and analyzed. These properties are 
examined by applying a bank of direction filters to the image in the Fourier domain. The direction in which the response is minimum is the corresponding blur direction. The directional filters could be modeled as a simple $1^{\text {st }}$ order filters or $2^{\text {nd }}$ order filter. These filters could also be modeled as masks of varying sizes and orientation (generally between 0 degrees and 360 degrees with a 1 degree increment). Figure 4.1 shows a general blockdiagram for estimation of the motion blur direction.

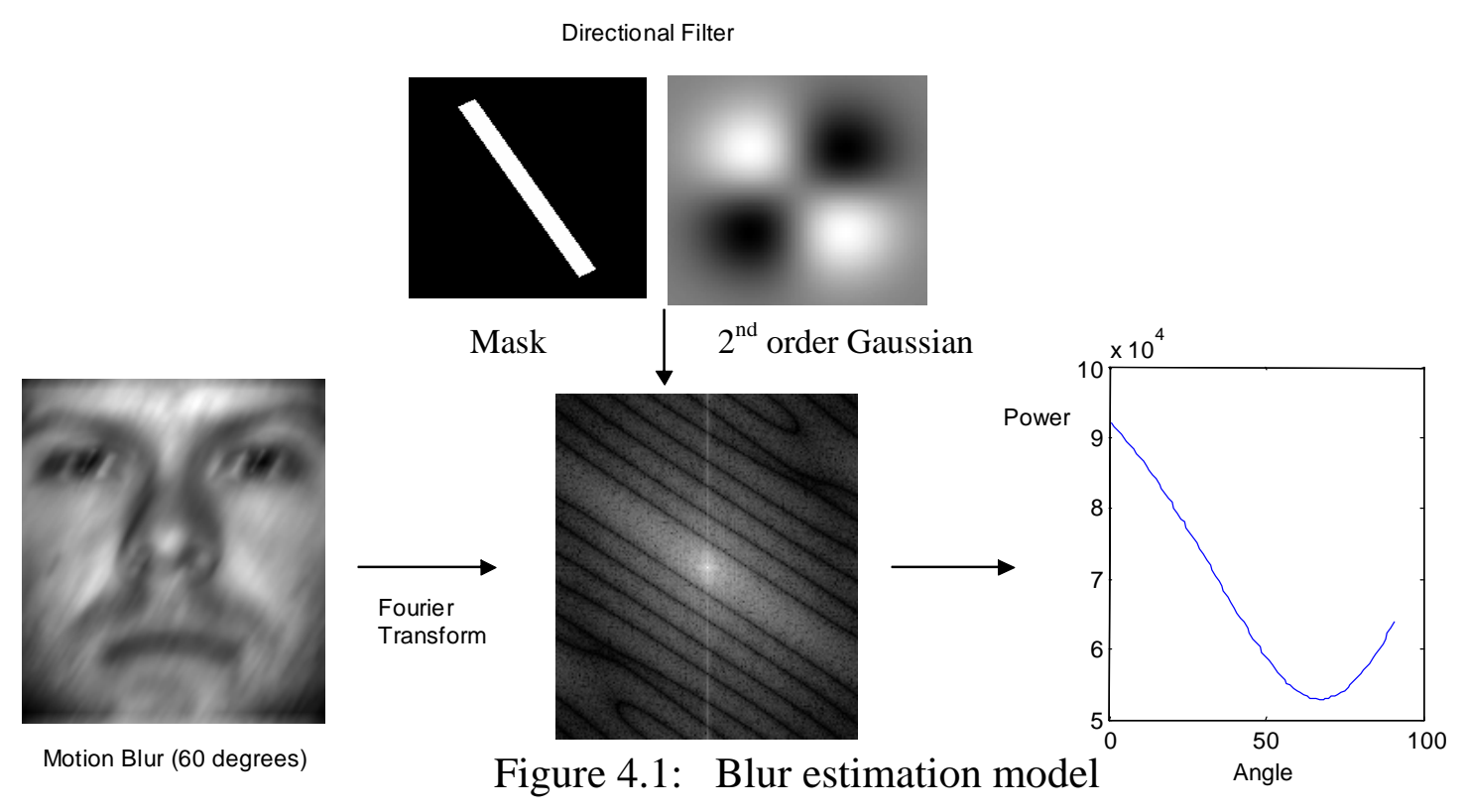

\section{Procedure:}

Given the blurred face image, we compute the two-dimensional Fourier transform of the image. The computation was done using the simple Matlab function "fft2”. As described earlier an image degraded by motion blur can be described linearly by

$$
I(x, y)=f(x, y) * h(x, y),
$$


where the function I represents the blurred image, function $\mathrm{f}$ represents the original image before blur and function h represents the PSF that causes the blur.

Based on the convolution theorem, the Fourier transform is computed as follows:

$$
F(I(x, y))=F(f(x, y)) \times F(h(x, y)),
$$

where $\mathrm{F}($.$) represents the Fourier transform.$

For the purpose of visualization we shift the resulting Fourier transform such that the zero frequency components are moved to the center of the resulting array. We further enhanced the image features by taking the logarithm of the shifted Fourier transform which has the property of magnifying minute differences while still keeping the zeros of the spectra [11]. The results of these operations are displayed in Fig 4.2. The directional filters are then applied in all orientations and the response computed. As previously stated, the orientation which produces the minimum response is the estimated direction of blur. Figure 4.3 displays two fourier transformed images and their corresponding response to a directional filter.

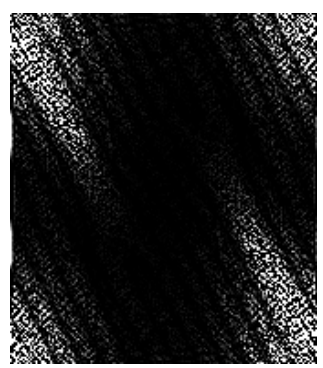

a) Image FT
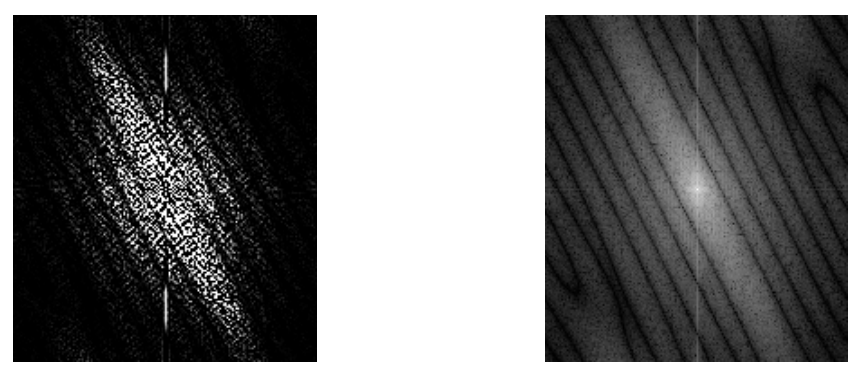

b) Shifted FT

c) Log of shifted FT

Figure 4.2: Fourier transform. 

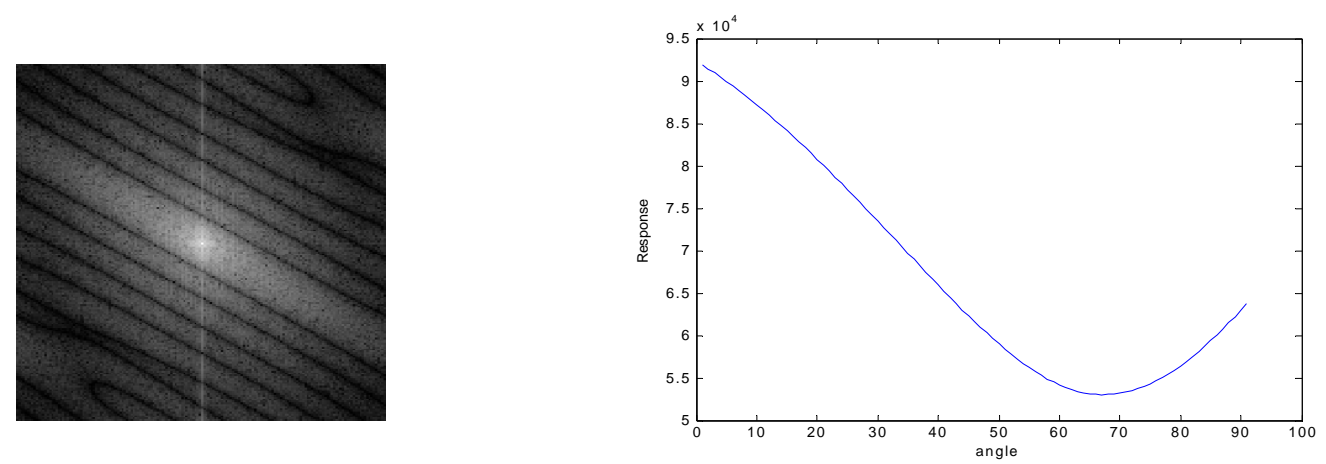

a)
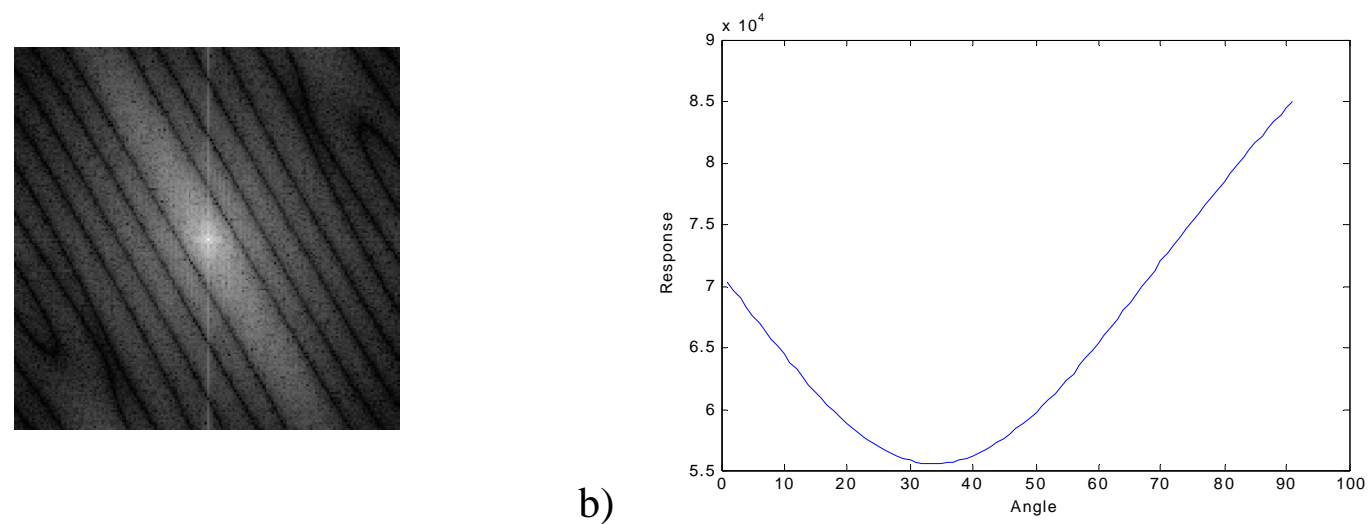

Figure 4.3: Image fft and corresponding filter response. (a) Blur angle $60^{\circ}$, (b) blur angle $30^{\circ}$

\subsubsection{Estimation of Blur strength}

Motion blur is analogous to stretching an elastic band. What happens when an image is blurred is that every point in the image is stretched and becomes a spread. In digital images these points are the pixel values. A spread of $\mathrm{x}$ corresponds to each pixel stretched over $\mathrm{x}$ pixels in the direction of the blur. This spread is evident from merely looking at a blurred image. The length of this spread resulting from motion blur corresponds to the strength of the blur. In lieu of this, blur strength can be estimated by simply estimating the length of the 
point spread caused by the blur. The length of this spread is estimated by computing the variance of the spread.

First we take the derivative of the blurred image in the direction of the blur in order to amplify the information contained in the image. Upon taking the derivative, we rotate the image in the direction of the estimated blur and compute the row covariance in the direction of the spread. This is why it was necessary that we compute the direction of blur first. The resulting covariance value is the blur strength.

Mathematically this procedure can be described as follows:

$$
\left[\begin{array}{l}
u^{\prime} \\
v^{\prime}
\end{array}\right]=\left[\begin{array}{cc}
\cos \theta & \sin \theta \\
-\sin \theta & \cos \theta
\end{array}\right]\left[\begin{array}{l}
u \\
v
\end{array}\right]
$$

1. Preprocess image to enhance hidden details ( Take the gradient)

2. Rotate the image by the estimated angle $\theta$

3. Compute the covariance in the horizontal direction

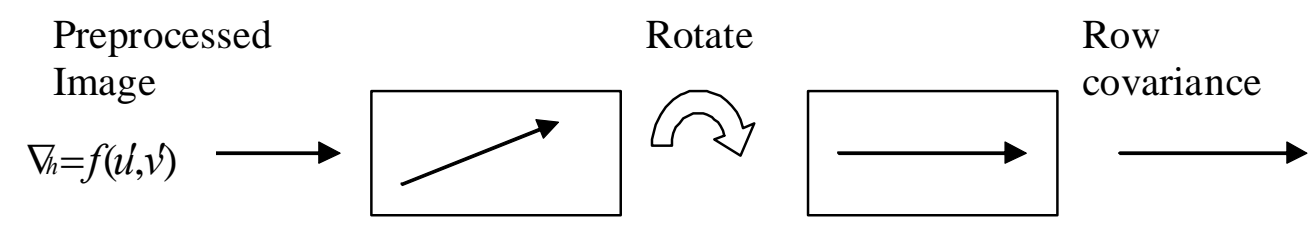

Figure 4.4: Block diagram for motion blur estimation 


\subsection{Defocus Blur}

There are a few methods already in existence to estimate defocused images. Majority of these methods can be applied to the face image with success.

One method is estimating the blur strength by measuring the amount of high frequency contents in the degraded image. The Daugman's filter [1] can be used to extract the high frequency content of the blurred image. We convolve the degraded image with the filter and compute the response (power). This power is normalized by the total power in the defocused image with 0 corresponding to an extremely poorly focused image and 1 corresponding to a perfectly focused image.

We propose another method to estimate defocus blur. This method is not been ascertained to be accurate for all types of blur and is still under scrutiny. The proposed method is similar to that of the motion blur discussed above. We proposed to estimate the strength of the blur by computing the covariance among the pixels of the blurred image. In this method as opposed to the motion blur method, the direction of blur is not a factor of defocus blur. With motion blur the derivative of the blurred image was computed in the direction of the blur. For defocus blur, we compute the horizontal derivative of the blurred images to magnify the blur and details not pronounced in the blurred image. The covariance of the image pixel values is computed and interpreted as an estimate of the blur strength. This method was tested on images synthetically degraded using the averaging filter. The size of the filter corresponds to the blur strength. We scaled the strength to better interpret the blur. A normalized value of 0 represents a very poorly defocused image and a value of 1 represents a perfectly focused 
image. Experimental results of the proposed method on synthetically degraded images are shown in Section 4.3.3.

\subsection{Lighting}

We estimate lighting by computing and comparing the entropy of the histogram of the face image. The terms histogram and entropy are described in sections 4.2.1 and 4.2.2 respectively. We describe how brightness can be estimated, how the direction of light can be estimated and how to estimate the degree of degradation. The next section briefly characterizes the properties of an image histogram

\subsubsection{Histogram}

A histogram gives information on the lighting of an image. It visually tells you if an image was properly exposed to light or not. Each pixel in a gray scale image has a brightness/darkness level called the gray level with discrete intensity values ranging from 0 to 255; 0 being the darkest and 255 being the lightest (white). The histogram displays these brightness levels [12]. The amplitude (value) of each level in the histogram is computed by counting Pixels with same grayscale (intensity) values. This count represents the value for that level in the histogram. The darker values represent shadows and the extreme lighter values represent highlights. An image histogram skewed to the right implies that the image has more dark pixels. The greater the skewness, the darker the image is, which in turn 
degrades face recognition. Image histogram skewed to the left depicts an image with a higher amount of bright pixels.

An image histogram also gives information on the contrast of an image. The thinner the histogram, the lower the contrast. Wider histograms typically have a better contrast. An image with a bad contrast will have its histogram compressed to one side.
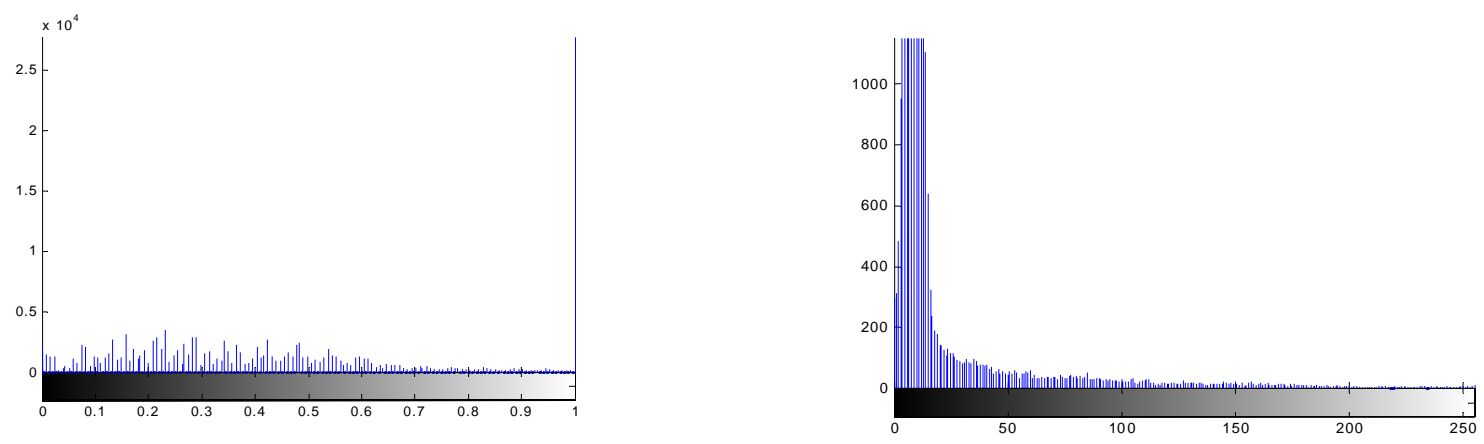

Figure 4.5: (Left) Histogram of an image with poor brightness, (Right) Histogram of an image with poor contrast

\subsubsection{Entropy}

Entropy measures the amount of information contained in an image [38]. In other words it measures the uncertainty of the intensity value distribution intensity. The entropy of an image is computed as follows:

$$
E\left(I_{p}\right)=-\sum_{k=0}^{N} \hat{p}(k) \log \hat{p}(k)
$$

where $\hat{p}(k)$ is the histogram (normalized) estimate of the image intensity density 


\subsubsection{Direction of External Light}

A face image can be subject to lighting from different directions. The lighting can be frontal, side or from the top, or from the back of an imaged individual. When a face image is illuminated by light, the illuminated part of the face would be relatively light compared to the other side of the face. The other parts would be relatively dark. If a light source illuminates a face from the right, the left side of the face will be darker compared to the right side of the face. The result of one side of a face image being darker or lighter than the other side is an uneven distribution of the pixel values which is apparent in the histogram distribution. The histogram of an image with even light distribution would be evenly distributed. On the other hand a face image with uneven pixel distribution would be unevenly distributed. The amount of dark or light pixels will depend on how close the light is. This property makes its easy to estimate the direction of a light source on a face image.

As discussed in section 4.2.1, the plot of the histogram of a face image depicts the distribution of pixels intensities. In lieu of this, the direction of a light is estimated by comparing the histogram (pixel distribution) of segments of the face.

The first step is to divide the face image into segments. We divide the image into four equal parts (segments) and compute the histogram of each part. The matlab function "imhist” was used to compute and plot the histogram. From visual inspection of the histograms, one can easily estimate the direction of light; the histograms would be skewed at different degrees. 
For quantitative estimation, we compute the skewness /entropy of each divided part of the face and compare values. The pair with the largest and highly significant difference tells us the direction of the illumination.
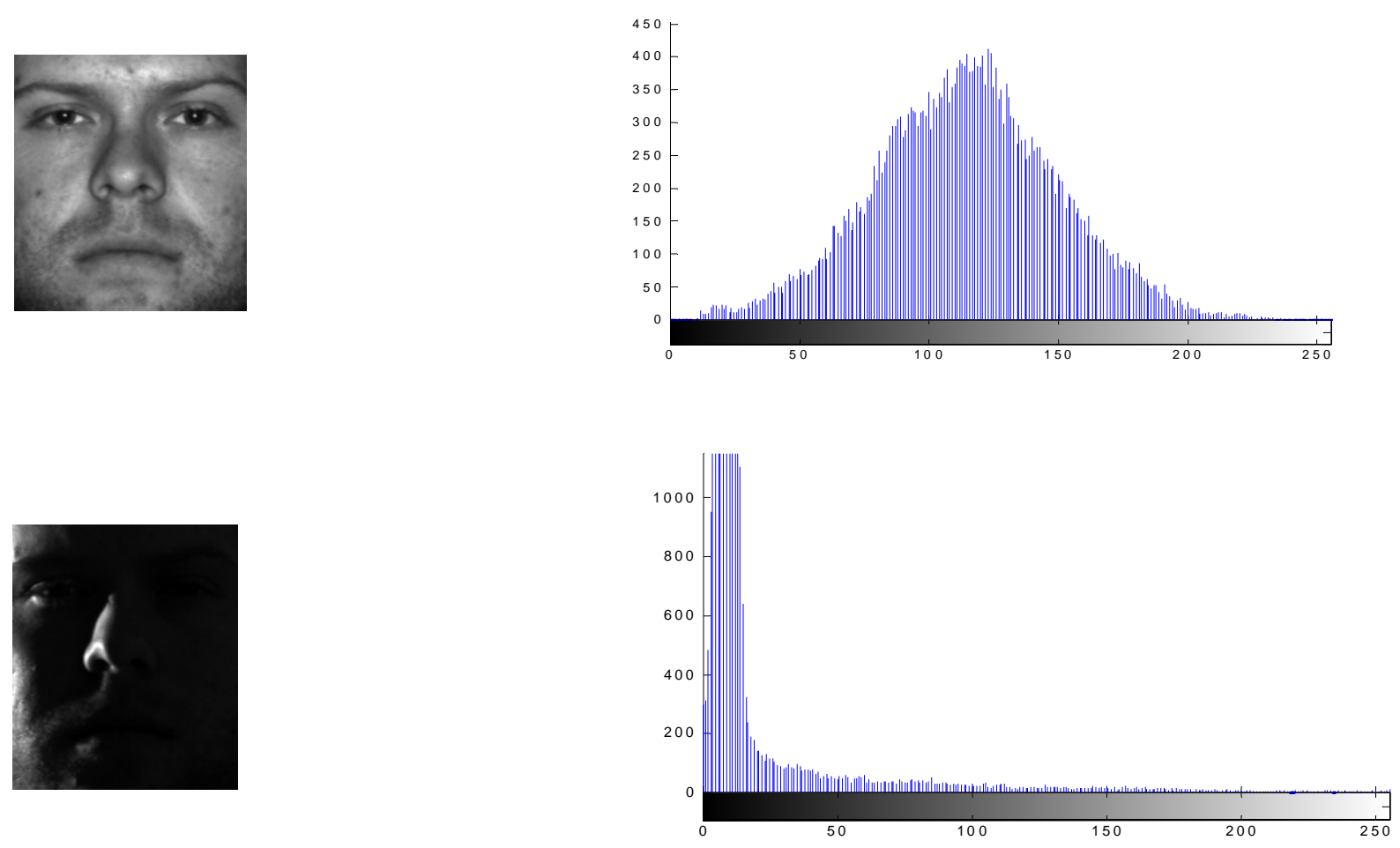

Figure 4.6: (Bottom) An image exposed to external light from the left of the subject

\subsubsection{Degree of degradation}

\section{Brightness}

Images with poor brightness generally contain less information. Because of the strong correlation between amount of information present in the image and performance, a face image with poor brightness would degrade recognition performance. As discussed in section 4.2.2, the entropy of an image measures the amount of information contained in the image. In 
lieu of this, the degree of brightness of a face image can be estimated by simply computing the entropy of the image. We compute the histogram first, from which we compute the image entropy.

\section{Uneven distribution due to External Lighting (Contrast)}

The degree of degradation resulting from the uneven distribution of information (intensity values) is estimated using the entropy feature of the histogram. The rationale behind this technique being that for an evenly distributed image, the mean intensity value is about the center of the image histogram, and hence the amount of information contained in the pixel values less than the mean intensity value would be approximately equal to the amount of information contained in the pixel values greater than the mean intensity value. We compute the mean intensity of the image and compute the entropy (see Section 4.32) of the intensity values lesser than the mean intensity and the intensity values greater than the mean intensity. The ratio of this entropy values give us an insight on the lighting distribution of the image.

Another technique to estimate the distribution of external light would be computing the relative entropy

We divide the face image segments (see Figure 4.7) and compute a normalized histogram of each segment. For each segment, we compute the ratio of the entropy of the intensity values less than 0.5 and the intensity values greater than 0.5 . We compute this ratio for all segments and compare values. An evenly distributed image would have similar values. 
For unevenly distributed images, the values would vary significantly depending on the degree of unevenness.

The higher this value the greater the degree of degradation.

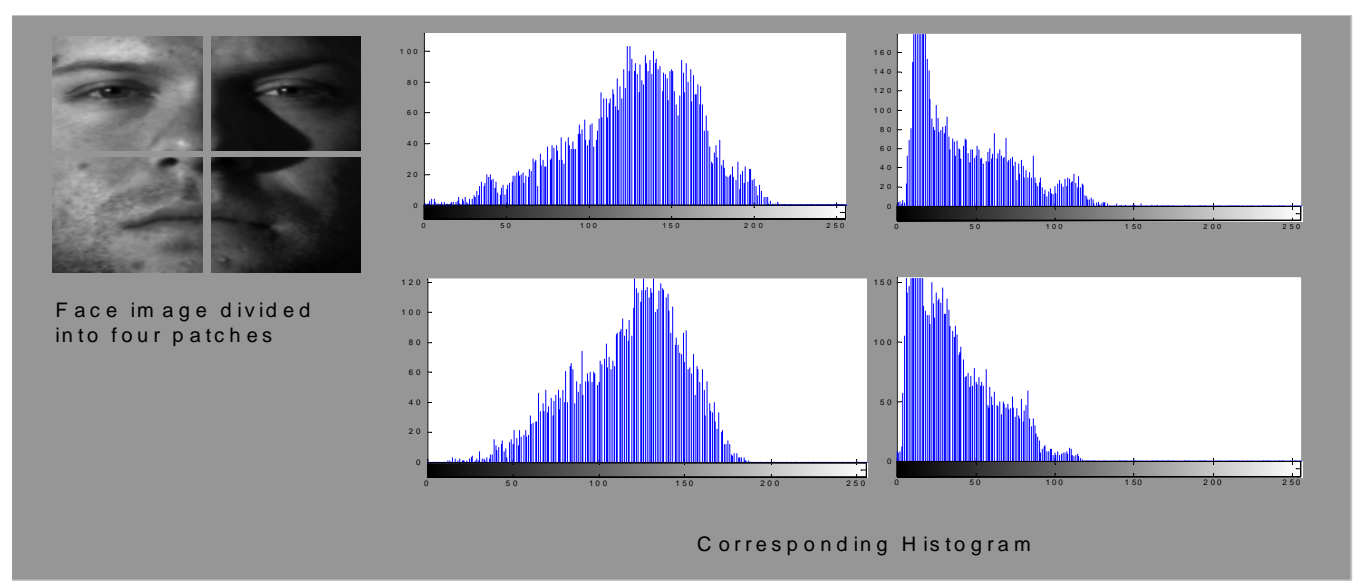

Figure 4.7: Histogram of four equally divided segments of a face image

\subsubsection{Experimental Result}

We tested the estimation techniques described in chapter 4 on synthetically degraded images and real life images (when available). Table 4.1 depicts the results for motion blur. It depicts the true direction, true strength and also the estimated direction and strength. Based on our results the technique seems to be fairly accurate. The more accurate the estimated direction of blur, the more accurate the estimated blur strength. The difference in the true blur orientation and the estimated blur orientation ranges between 0 and 8 degrees while the difference in the true blur strength and the estimated blur strength ranges between 0 and 3 pixels. 
Figure 4.8 and Table 4.2 depicts the experimental results for defocus blur. Figure 4.8 shows the experimental results from using the first estimation method - Convolving the degraded image with Daugman's filter and measuring the frequency content. One corresponds to perfect focus and zero corresponds to a very poorly focused image. Table 4.2 shows the experimental results from using the second proposed estimation technique Computing the row covariance. This method was tested on images synthetically degraded using averaging filter. The strengths correspond to the size of the filter.

\begin{tabular}{|c|c|c|c|c|}
\hline $\begin{array}{c}\text { Type of } \\
\text { Blur }\end{array}$ & $\begin{array}{c}\text { True } \\
\text { Direction } \\
\text { (deg) }\end{array}$ & $\begin{array}{c}\text { True } \\
\text { Strength } \\
\text { (pixels) }\end{array}$ & $\begin{array}{c}\text { Estimated } \\
\text { Direction } \\
\text { (deg) }\end{array}$ & $\begin{array}{c}\text { Estimated } \\
\text { Strength } \\
\text { (pixels) }\end{array}$ \\
\hline Synthetic & 0 & 7 & 0 & 6 \\
\hline Synthetic & 0 & 10 & 0 & 9 \\
\hline Synthetic & 0 & 15 & 0 & 14 \\
\hline Synthetic & 15 & 7 & 11 & 7 \\
\hline Synthetic & 15 & 10 & 11 & 9 \\
\hline Synthetic & 15 & 15 & 10 & 14 \\
\hline Synthetic & 30 & 7 & 23 & 7 \\
\hline Synthetic & 30 & 10 & 24 & 14 \\
\hline Synthetic & 30 & 15 & 24 & 7 \\
\hline Synthetic & 45 & 7 & 38 & 10 \\
\hline Synthetic & 45 & 10 & 39 & 7 \\
\hline Synthetic & 45 & 15 & 39 & 10 \\
\hline Synthetic & 60 & 7 & 59 & 14 \\
\hline Synthetic & 60 & 10 & 58 & 6 \\
\hline Synthetic & 60 & 15 & 57 & 14 \\
\hline Synthetic & 90 & 7 & 90 & 11 \\
\hline Synthetic & 90 & 10 & 90 & 2 \\
\hline Synthetic & 90 & 15 & 90 & \\
\hline Real & N/A & N/A & 150 & 20 \\
\hline Real & N/A & N/A & & 9 \\
\hline
\end{tabular}

Table 4.1: Motion blur experimental result 


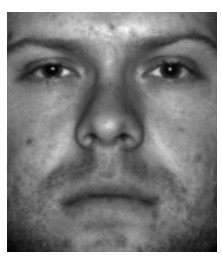

Original image

Focus $=0.98$

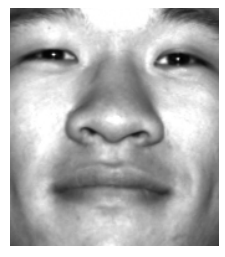

Original image

Focus $=0.96$

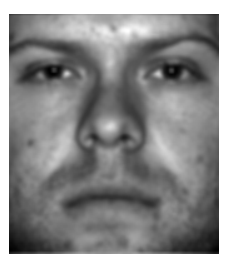

Filter size $=4 \times 4$

Focus $=0.94$

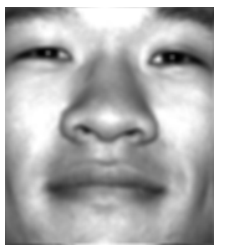

Filter size $=4 \times 4$

Focus $=0.89$

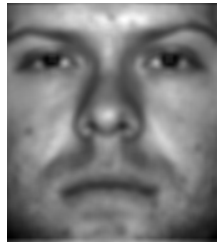

Filter size $=6 \times 6$

Focus $=0.85$

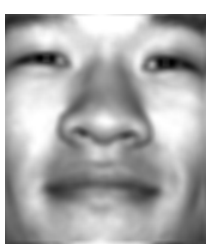

Filter size $=6 \times 6$

Focus $=0.75$

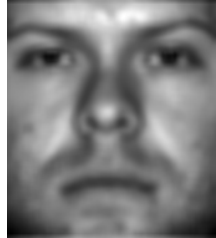

Filter size $=8 \times 8$

Focus $=0.67$

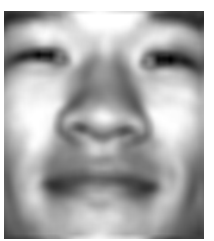

Filter size $=8 \times 8$

Focus $=0.52$

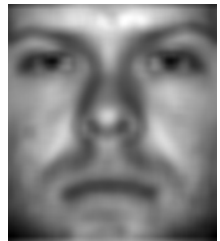

Filter size $=10 \times 10$

Focus $=0.46$

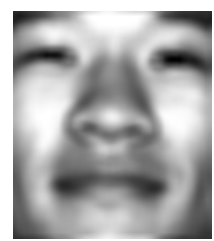

Filter size $=10 \times 10$

Focus $=0.38$

Figure 4.8: Defocus blur experimental result using method 1

\begin{tabular}{|lrr}
\hline \multicolumn{1}{|c}{ Type of Blur } & True Kernel size & $\begin{array}{c}\text { Estimated } \\
\text { Strength }\end{array}$ \\
\hline Synthetic & 2 & 3 \\
Synthetic & 10 & 10 \\
Synthetic & 15 & 15 \\
Synthetic & 20 & 19 \\
Synthetic & 25 & 24 \\
Synthetic & 40 & 39 \\
\hline
\end{tabular}

Table 4.2: Defocus blur experimental result using method 2

The experimental results for lighting are depicted below (Figure 4.9). The further away the mean intensity of the image is to 0.5 the greater the computed ratio and the greater this ratio, the worse the image contrast. Figure 4.10 show the ROC curves for lighting for both the eigenface and eigenphase algorithm. FRR stands for false reject rate while FAR stands for false accept rate. 

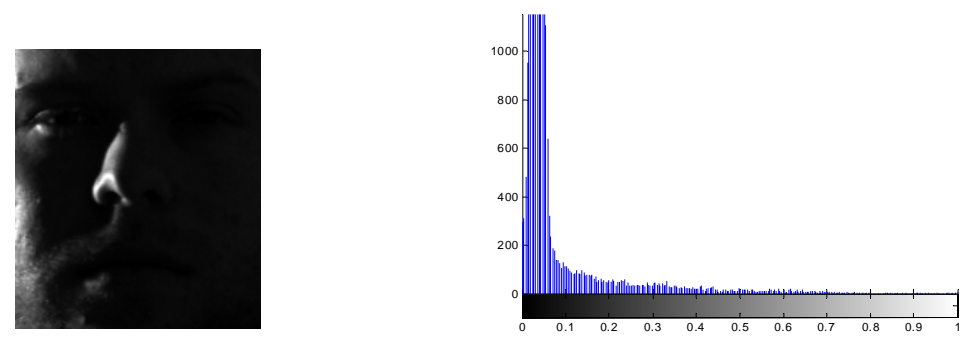

$$
\text { Mean }=0.0818
$$

Ratio $=43.72$

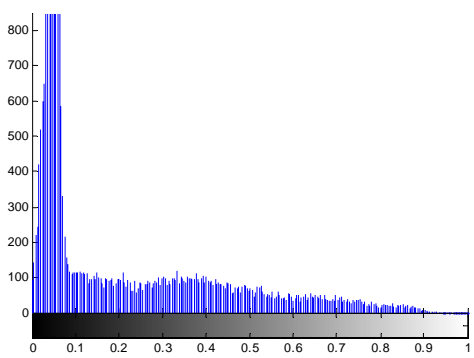

$$
\text { Mean }=0.1863
$$

Ratio $=6.93$

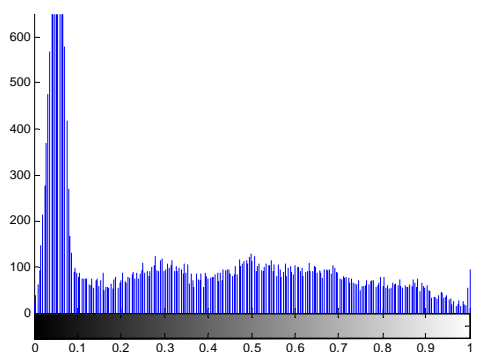

Mean $=0.3069$

Ratio $=2.69$
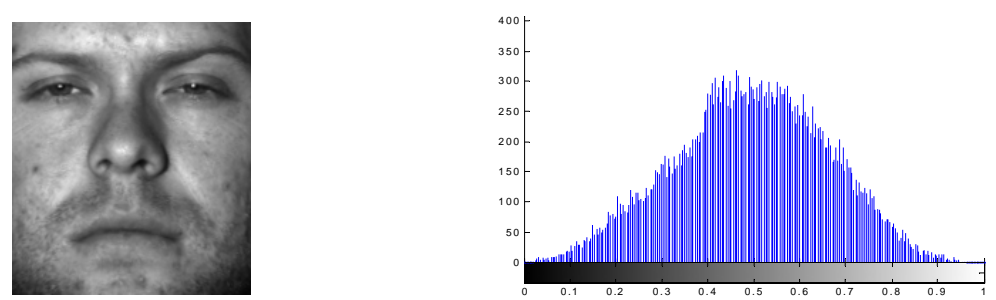

Mean $=0.4943$

Ratio=1.07

Figure 4.9: Experimental results for lighting variation (contrast) 

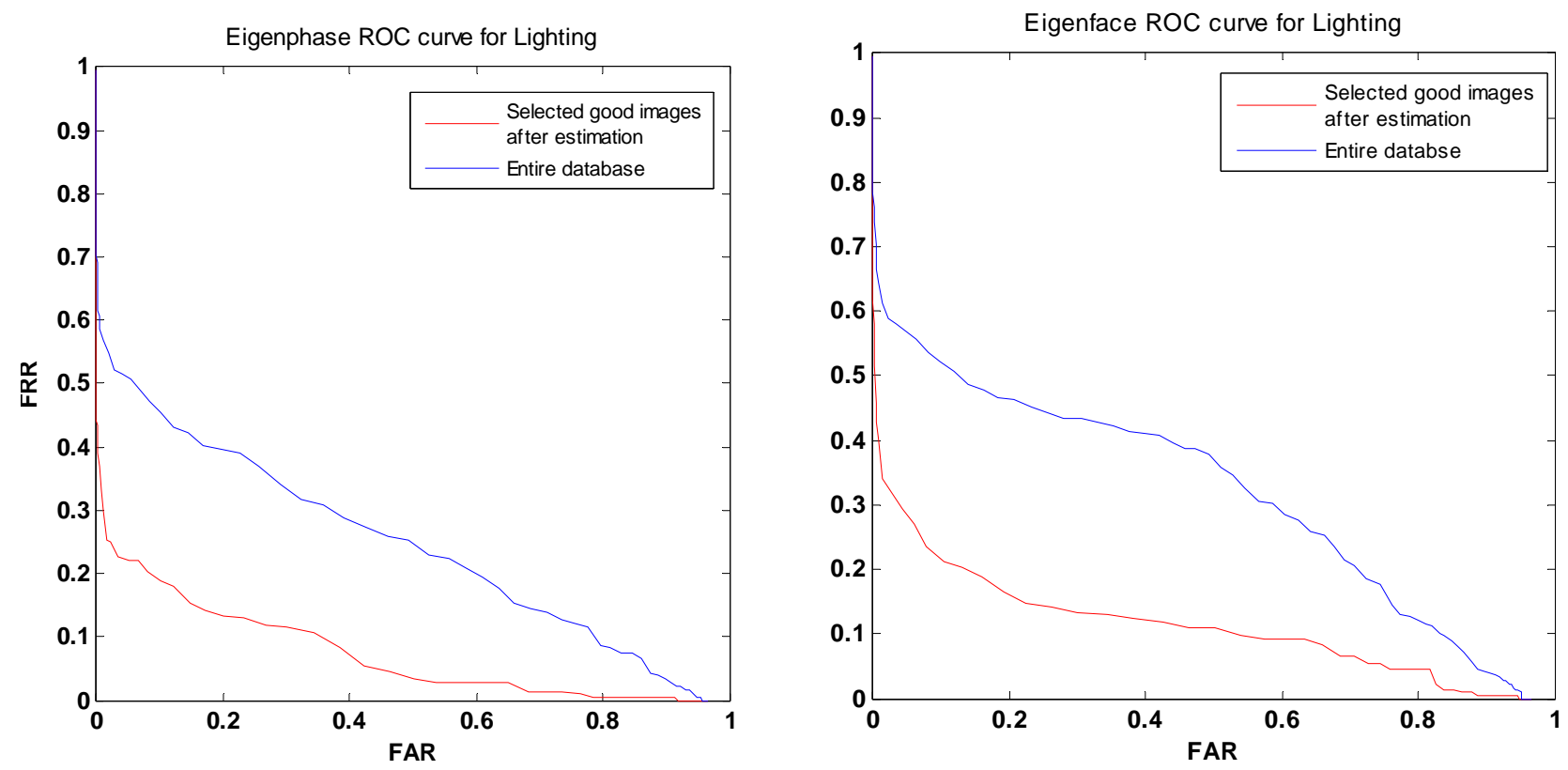

Figure 4.10: ROC curves for lighting variation (contrast), left (Eigenphase), right (Eigenface) 


\section{Chapter 5}

\section{Conclusions}

Image quality degrades the performance of face recognition algorithms. In this thesis we listed and discussed quality factors that degrade a face image and ultimately recognition performance. The effects of these image quality factors on the eigenface and eigenphase algorithm were studied using the Genuine and Imposter Euclidean distance Scores.

Of all quality factors studied, Lighting tends to degrade recognition performance the most, followed by occlusion. Motion blur and defocus blur were also studied to also degrade recognition performance with a higher effect on the eigenphase face recognition method. Pixel Count and cropping on the other hand do not seem to affect the recognition algorithms discussed in this thesis.

We also proposed and discussed algorithms to estimate three of the image quality factors including motion blur, defocus blur and lighting.

Lighting (Brightness and Contrast) was estimated using the entropy feature. Motion blur and defocus blur on the other hand were estimated by computing the covariance of the image pixels. 


\section{Chapter 6}

\section{Future Work}

1. In this thesis we studied the effect of image quality factors on face recognition using only the Eigenface and Eignenphase algorithms. The effect of this quality factors may vary for different face recognition algorithms. In future work, we propose a study that includes a number of state-of-the-art face recognition methods.

2. In this work, estimation was discussed for 3 of the image quality factors. In future work, we propose to design estimation techniques to evaluate other image quality factors not estimated in this thesis.

3. The effects of Pose and age were not discussed in this thesis. We propose to study the effects of these factors and algorithms to estimate them.

4. Further study could be conducted to propose recognition algorithms invariant to the factors discussed in this thesis

5. In this work, we assumed that the face image is degraded by one quality factor at a time. In practice a combination of this factors degrade image quality simultaneously. In future work we propose a joint study and estimation of image quality factors. 


\section{References}

[1] J. Daugman. "How Iris Recognition Works” in proc IEEE Transaction on Circuits and Systems for Video Technology, 14(1), 2004, pp. 21-30

[2] Jon Krueger, Marshall Robinson, Doug Kochelek, Matthew Escarra, “Obtaining the Eigenface basis”, Retrieved from http://cnx.rice.edu/content/m12531/latest/

[3] M. Turk, A. Pentland, “Eigenfaces for Recognition”, Journal of Cognitive Neuroscience. Vol 3, No. 1. 71-86, 1991. Retreived from Dimitri Pissarenko, Eigenface based facial recognition, 2003, http://openbio.sourceforge.net/resources/eigenfaces/eigenfaceshtml/facesOptions.html

[4] http://en.wikipedia.org/wiki/Eigenface.

[5] M. Kirby and L. Sirovich (1990). Application of the Karhunen-Loeve procedure for the characterization of human faces, IEEE Transactions on Pattern analysis and Machine Intelligence, 12(1): 103-108

[6] Alex Rav-Acha, Shmuel Peleg, "Restoration of Multiple Images with Motion Blur in Different Directions" proc. of the Fifth IEEE Workshop on Apllications of Computer Vision, 2000, pp. 22-28

[7] John D. Woodward, jr., Christopher Horn, Julius Gatune, Aryn Thomas, "Biometrics: A Look at Facial Recognition", Rand 2003 [Electronic version]. Retrieved from http://www.rand.org/pubs/documented_briefings/2005/DB396.pdf

[8] Samir Nanavati, Michael Thieme, Raj Nanavati (2002). Biometrics: Identity verification in a Networked World. John Wiley and Sons, Inc. 
[9] Adrian Stern, Inna Kruchakov, Eitan Yoavi, Norman S. Kopeika, "Recognition of motion-blurred images by use of the method of moments” Applied Optics, Vol. 41, No. 11, April 2002, pp. 2164-2171

[10] Y. Yitzhaky, N.S Kopeika, "Identification of Motion Blur for Blind Image Restoration” IEEE symposium in Tel-Aviv, March 1995

[11] Loannis Rekleitis, “Visual Motion Estimation based on Motion Blur Interpretation”. M.Sc thesis, School of Computer Science, McGill University, 1995

[12] Understanding Histograms. Retrieved from http://www.luminous landscape.com/tutorials/understanding-series/understanding-histograms.shtml

[13] Matthew A. Turk, Alex P. "Pentland, Face Recogniton using Eigenfaces”, In proc of the IEEE Conference on Computer Vision and Pattern Recognition, pages 586-591, Maui, Hawaii, Dec 1991,

[14] L. Ma, Y. Wang. T. Tan, “Iris Recognition Using Circular Symmetric Filters”, the sixteenth International Conference on Pattern Recognition, Vol. II, pp. 414-417, 2002.

[15] N.D. Kalka, J. Zuo, V. Dorairaj, N.A. Schmid and B. Cukic, Image Quality Assesment for Iris Biometric, submitted for presentation at the SPIE 2005.

[16] Lizuo Jin, Shin’ichi Satoh, Massao Sakauchi, A novel Adaptive Enhancement Algorithm for Face Detection, in proc. of the $17^{\text {th }}$ International Conference on Pattern Recognition, 2004, pp. 1051- 4651

[17] Georghiades, A. S, Belhumer, P.N, Kriegman, D.J, "From Few to many, Illumination Cone Models for Face Recognition under Variable Lighting and Pose” In IEEE 
Transaction on Pattern Analysis and Machine Intelligence, vol. 23(6), 2001, pp. 643660.

[18] Alex (Sandy) Pentland, Tanzeem Choudry. "Face Recognition for Smart Environments," computer, vol. 33, no. 2, pp 50 -55

[19] Y. Yitzhaky, N.S Kopeika, Identification of Blur Extent from Motion Blurred Images, SPIE vol. 2470, pp. 1-11

[20] Young-Ouk Kim, Joonki Paik, Jingu Heo, Anfreas Koschan, Besma Abidi, Mongi Abidi, Automatic Face Recognition Tracking for Highly Accurate Face Recognition in Uncobstarined Environments, 2005

[21] A.U. Batur, B. E Flinchbaugh, M. H Hayes III, A DSP-Based Approach for the Implementation of Face Recognition Algorithms, ICASSP 2003

[22] Yongsheng Gao, Maylor K.H. Leung, "Face Recogniton Using Line Edge Map” in IEEE Transaction on Pattern Analysis and Machine Intelligence, vol 24, No. 6, June 2002, pp. 764-773.

[23] J. Heo, B. Abidi, J. Paik, M. A. Abidi "Face Recognition: evaluation report for FaceIt", proc of the SPIE $6^{\text {th }}$ International Conference on Quality Control by Artificial Vision, Vol. 5132, May 2003, PP. 551-558.

[24] Marios Savvides, B.V.K. Vijaya Kumar and P.K., Khosla, "Eigenphases vs. Eigenfaces". Proc. of the $17^{\text {th }}$ International Conference on Pattern Recognition, vol. 23, August 2004 pp. 810-813

[25] Matthew A. Turk, Alex P. "Pentland, Face Recogniton using Eigenfaces”, In proc of the IEEE Conference on Computer Vision and Pattern Recognition, Maui, Hawaii, December 1991, pp. 586-591. 
[26] C. Mayntz, T. Aach, D. Kunz, "Blur Identification using a Spectral Inertia Tensor and Spectral Zeros”, proc. of the 1999 International Conference on Pattern Recognition, vol. 2, 1999, pp. 885-889

[27] Y. Yitzhaky, N.S. Kopeika, Evaluation of the Blur Parameters from Motion Blurred Images, IEEE, 1996, pp. 216-219

[28] Lizuo Jin, Shin’ichi Satoh, Massao Sakauchi, “A novel Adaptive Image Enhancement Algorithm for Face Detection" in proc. of the $17^{\text {th }}$ International Conference on Pattern Recognition, 2004, pp. 1041- 4651.

[29] Adrian Stern, Inna Kruchakov, Eitan Yoavi, Norman S. Kopeika, "Recognition of Motion-blurred Images by use of the Methods of Moments” in Applied Optics, vol 41, No. 11, April 2002, pp 2164 -2171.

[30] W. Zhao, R. Chellappa, P.J. Phillips, A. Rosenfeld, Face Recognition: A Literature Survey, ACM computing surveys, Vol. 35, No. 4, December 2003, pp. 399-458.

[31] Yael Adini, Yael Moses, Shimon Ullman, Face Recognition: "The problem of compensating for changes in illumination Direction”, in IEEE Transaction on Pattern Analysis and Machine Intelligence, Vol 19, No. 7, July 1997, pp. 721-732.

[32] Paul Griffin, Understanding the Face Image Format Standards, Identix, April 2005, pp. $1-31$

[33] Qing Jiang, “Principal Component Analysis and Neural Network Based Face Recognition”. M.Sc thesis, Retrieved from http://people.cs.uchicago.edu/ qingj/ThesisHtml

[34] LinLin Shen, Li Bai, “Gabor Feature Based Face Recognition using Kernel Methods” ”, in IEEE International Conference on Automatic Face and Gesture Recognition, Vol 19, No. 7, July 1997, pp. 721-732. 
[35] Stan Z. Li, Anil K. Jain. (2004). Handbook of Face Recognition. New York: Springer Science+Business Media.

[36] A. M. Tekalp, H. Kaufman, J. Woods, Identification of Image and Blur Parameters for the Restoration of Non Causal Blurs, IEEE Transaction, Acoust, Speech and Signal Processing, Vol. ASSP-34, pp. 963-972, 1986

[37] A. K Katsaggelos, K. T. Lay, Maximum Likelihood Identification and Restoration of Images Using Expectation-Maximization Algorithm, Digital Image Restoration, A.k Katsaggelos (Ed), Springer-Verlag, 1991, pp. 143-176.

[38] Anil K. Jain. (1989). Fundamentals of Digital Image Processing. New Jersey: PrenticeHall, Inc

[39] A. V. Oppenheim and J.S Lim. The importance of Phase in signals. Proc. IEEE, Vol 69, No. 5, May 1981, pp. 529-541 